\title{
Homotopy characterization of non-Hermitian Hamiltonians
}

\author{
Charles C. Wojcik $\odot,{ }^{1}$ Xiao-Qi Sun, ${ }^{2,3}$ Tomáš Bzdušek, ${ }^{2,4,5}$ and Shanhui Fan ${ }^{1, *}$ \\ ${ }^{1}$ Department of Electrical Engineering, Ginzton Laboratory, Stanford University, Stanford, California 94305, USA \\ ${ }^{2}$ Department of Physics, McCullough Building, Stanford University, Stanford, California 94305, USA \\ ${ }^{3}$ Stanford Center for Topological Quantum Physics, Stanford University, Stanford, California 94305, USA \\ ${ }^{4}$ Condensed Matter Theory Group, Paul Scherrer Institute, CH-5232 Villigen PSI, Switzerland \\ ${ }^{5}$ Department of Physics, University of Zürich, 8057 Zürich, Switzerland
}

(Received 1 January 2020; revised manuscript received 9 April 2020; accepted 22 April 2020; published 15 May 2020)

\begin{abstract}
We revisit the problem of classifying topological band structures in non-Hermitian systems. Recently, a solution has been proposed, which is based on redefining the notion of energy band gap in two different ways, leading to the so-called "point-gap" and "line-gap" schemes. However, simple Hamiltonians without band degeneracies can be constructed which correspond to neither of the two schemes. Here, we resolve this shortcoming of the existing classifications by developing the most general topological characterization of non-Hermitian bands for systems without a symmetry. Our approach, which is based on homotopy theory, makes no particular assumptions on the band gap, and predicts significant extensions to the previous classification frameworks. In particular, we show that the one-dimensional invariant generalizes from $\mathbb{Z}$ winding number to the non-Abelian braid group, and that depending on the braid group invariants, the two-dimensional invariants can be cyclic groups $\mathbb{Z}_{n}$ (rather than $\mathbb{Z}$ Chern number). We interpret these results in terms of a correspondence with gapless systems, and we illustrate them in terms of analogies with other problems in band topology, namely, the fragile topological invariants in Hermitian systems and the topological defects and textures of nematic liquids.
\end{abstract}

DOI: 10.1103/PhysRevB.101.205417

\section{INTRODUCTION}

Topological invariants associated with energy bands in the reciprocal momentum $(\boldsymbol{k})$ space have proven useful in predicting novel physical phenomena [1,2], including robust unidirectional transport, in both electronic and photonic systems. Examples of topological invariants include Chern numbers [3], which are defined for general systems lacking any particular symmetry, as well as $\mathbb{Z}_{2}$ invariants [4] and winding numbers [5], which are defined as long as some symmetry is preserved. Classification schemes such as the tenfold way [6-8] and its various extensions [9] provide a unified approach within the mathematical frameworks of $K$ theory and homotopy theory and enable a systematic understanding of the implications of different symmetries for topological invariants.

Non-Hermitian Hamiltonians have widespread applications in describing open systems. For example, the ubiquitous loss and gain in photonic systems [10-32], the finite quasiparticle lifetimes [33-37], and certain statistical-mechanical models [38] are naturally described in terms of non-Hermitian Hamiltonians. Recently, there has been a growing interest in uncovering novel topological phases in non-Hermitian systems [39-61]. Although these questions have been partially addressed in theory $[42,62,63]$, a unified mathematical description of non-Hermitian band topology is still lacking, even for the most basic setting when no symmetry is as-

*Corresponding author: shanhui@stanford.edu sumed. This is most manifest in the innate dichotomy of the recently suggested $K$-theory classification framework, which distinguishes two schemes, the "line-gap" and the "point-gap" schemes $[25,62]$. Within the line-gap scheme, the complex energy spectrum is assumed to miss a line in the complex plane. This allows one to deform the Hamiltonian into one which is Hermitian with no symmetries (class A), implying integer topological invariant in even spatial dimensions. In contrast, within the point-gap scheme, the complex energy spectrum is assumed to miss a point in the complex plane. This facilitates a continuous deformation into a Hermitian Hamiltonian with chiral symmetry (class AIII), and implies integer topological invariants in odd spatial dimensions.

However, there are interesting topologically nontrivial nonHermitian Hamiltonians that are not uniquely characterized by either a point gap or a line gap. A prototypical example of a such a Hamiltonian is an exceptional ring [54], which arises when a generic non-Hermitian perturbation is applied to a Weyl point [27,64]. Although our focus in this paper is on gapped systems, the gapless exceptional ring provides a vivid illustration of the difficulty of separately considering pointand line-gap classifications. The exceptional ring carries both a one-dimensional (1D) and a two-dimensional (2D) invariant simultaneously, depending on which type of gap one considers. Curiously, the two invariants have non-trivial influence on each other and therefore cannot always be decoupled. Especially, Ref. [65] showed that in non-Hermitian systems with exceptional lines, the Chern number of an exceptional ring ceases to be conserved, but can change sign through a reciprocal braiding process [65-68]. This observation 
suggests the need for a more general classification framework, which does not make any assumption on the band gap being globally a point vs a line, and which naturally incorporates the interaction between the invariants defined on manifolds with various spatial dimensions.

In this paper, we develop such a unified classification using homotopy theory. Within a concise mathematical framework, we revisit the derivation of $1 \mathrm{D}$ and $2 \mathrm{D}$ topological invariants of non-Hermitian bands. As opposed to the two $K$-theory classification schemes described above, our homotopy theory approach is also sensitive to unstable topological invariants [69]. Our derivation significantly extends the previous theoretical works $[42,62,63]$ and explicitly captures the interaction of these invariants. Specifically, the theory allows us to classify non-Hermitian Hamiltonians defined on a periodic 2D $\boldsymbol{k}$ space, with the surprising result that depending on the braid-group-valued 1D invariants, the $\mathbb{Z}$-valued Chern numbers can be replaced by $\mathbb{Z}_{n}$-valued invariants, where the value of $n$ depends on the details of the cycle type of the braids along the Brillouin zone torus. We note that the interaction of topological invariants in various dimensions is an intrinsically non-Hermitian phenomenon, which is inaccessible in perturbative approaches which start from Hermitian models. As we explain, this interaction is captured mathematically by the action of $\pi_{1}$ on $\pi_{2}$ [70], a classical piece of homotopytheoretic data which is absent in classifications based on the tenfold way, but crucial in our non-Hermitian classification scheme.

The paper is organized as follows. The first two sections present the classification result in a detailed, pedagogical manner. First, in Sec. II, we revisit the derivation of the topological invariant for two-band Hermitian systems. The goal is to reformulate this simple story using a mathematical language that is more appropriate for the generalization to non-Hermitian systems, which constitute the contents of Sec. III. In this section, we highlight the uniqueness which arises in the nonHermitian setting, both in terms of rigorous mathematics and intuitive pictures. Next, in Sec. IV, we describe the interaction between 1D and 2D invariants as reported for gapless non-Hermitian systems in Ref. [65], and explain how this interaction relates to the modified topological classification of gapped systems. To further extend the intuition for this classification, we also report on certain relationships with the physics of nematic liquids [71-73] and with the fragile topology of real-symmetric Bloch Hamiltonians [66-68,7476], which provide a useful analogy for understanding the topology of non-Hermitian Bloch Hamiltonians. We also provide a calculation of these invariants in an explicit model. Finally, in Sec. V, we generalize these results to many bands, finding braid group and $\mathbb{Z}_{N}$ invariants.

\section{TOPOLOGICAL CLASSIFICATION OF TWO-BAND HERMITIAN HAMILTONIANS}

The idea of topology has been prominent in the study of electronic band structures in the last few decades. This notion emerges naturally from the ultimate task of condensed matter physics: to classify and discover phases of matter and study phase transitions. At the phase-transition critical point, many systems show scale-invariant characteristics, indicating that there is no finite characteristic length or energy scale at low energy. For a noninteracting electron problem, this point corresponds to a gapless band structure. Therefore, a phase transition corresponds to a gap-closing process. The notion of topology is essential to describing phases: as long as the continuous tuning of the Hamiltonian does not result in a gap closing, the system remains in the same phase. Studying the equivalence classes of Hamiltonians under continuous tuning without closing a gap therefore becomes a problem in topology, or more specifically homotopy theory.

In this section, we review this classification problem using a two-band example in 2D, in a formalism that can be generalized to non-Hermitian cases. We split our presentation into four subsections. In the first subsection, we introduce notation and define the classification problem. The main objects of interest which we introduce are a topological space $X$ (here it is the space of Hermitian Hamiltonians with a spectral gap) and a set $\left[T^{2}, X\right]$ (equivalence classes of such Hamiltonians defined on a Brillouin zone torus $T^{2}$ ). In the second subsection, we develop a characterization of the space $X$. The main result here is that $X$ is homeomorphic to the 2-Bloch sphere $S^{2}$, i.e., $X \sim S^{2}$. In the third subsection, we compute the set $\left[T^{2}, X\right]$ and find $\left[T^{2}, X\right]=\mathbb{Z}$. This is done in several steps, the first of which is computing the homotopy groups $\pi_{n}(X)$ (equivalence classes of gapped Hamiltonians defined on an $n$-sphere $S^{n}$, representing strong topological invariants of various dimensions). In the final subsection, we define an action of $\pi_{1}(X)$ on $\pi_{2}(X)$, which will be of crucial importance in the non-Hermitian setting.

\section{A. Defining the classification problem}

For simplicity, we consider a two-band Hamiltonian describing a 2D lattice model. In momentum space, the Bloch Hamiltonian is simply a family of $2 \times 2$ Hermitian matrices $\mathcal{H}(\boldsymbol{k})$, where $\boldsymbol{k}$ ranges over the wave vectors in the $2 \mathrm{D}$ first Brillouin zone. Because $\mathcal{H}(\boldsymbol{k})$ is periodic in both directions, we can identify opposite edges of the first Brillouin zone and consider the wave vector $k$ as a point in a torus $T^{2}$. Then, $\mathcal{H}(\boldsymbol{k})$ defines a continuous map $\mathcal{H}: T^{2} \rightarrow \operatorname{Herm}_{2}(\mathbb{C})$ from the momentum-space torus to the set of Hermitian $2 \times 2$ matrices. Furthermore, as we motivate from the notion of a topological phase transition, we are interested in the equivalence classes of the Hamiltonians upon continuous deformation without gap closing. Therefore, the space we classfiy is the more restricted space $X$, which is the set of gapped $2 \times 2$ Hermitian matrices, i.e., those with distinct eigenvalues. This can be equivalently and concisely formulated by requiring that the discriminant, defined as $\operatorname{Disc}(\mathcal{H})=\prod_{i<j}\left(\lambda_{i}-\lambda_{j}\right)^{2}$ [where $\left\{\lambda_{i}\right\}_{i=1}^{\operatorname{dim}(\mathcal{H})}$ are the eigenvalues of $\mathcal{H}]$, is nonvanishing for the Hamiltonian. Therefore, we define our target space of gapped Hamiltonians as

$$
X:=\left\{\mathcal{H} \in \operatorname{Herm}_{2}(\mathbb{C}): \operatorname{Disc}(\mathcal{H}) \neq 0\right\} .
$$

Notably, $\operatorname{Disc}(\mathcal{H})$ is a polynomial in the coefficients of $\mathcal{H}$, so $X$ is the complement of a hypersurface inside a fourdimensional (4D) vector space and can therefore be expected to be topologically interesting. Then, $\left[T^{2}, X\right]$ is the set of homotopy classes of Hamiltonians which have distinct energies at every point in momentum space. Topological classification 
with this choice of $X$ means that topological invariants can change under continuous deformations of $\mathcal{H}(\boldsymbol{k})$, but specifically only under those which close the spectral gap (which correspond to phase transitions).

In the Hermitian case, our gap condition agrees with the standard one in the literature. In the non-Hermitian case in Sec. III, we will use the same gap condition. There, this definition will not be equivalent to either the point-gap or linegap conditions used in Ref. [62]. It is, however, equivalent to the definition of separable bands from Ref. [39].

\section{B. Characterizing the target space}

We now need to characterize $X$ in a way that makes its topological structure more apparent. We do this by parametrizing $X$ in terms of eigenvectors and eigenvalues, performing an eigendecomposition. This allows us to describe $X$ in terms of more familiar topological spaces. According to the spectral theorem, the eigenvectors of a Hermitian matrix $\mathcal{H}$ constitute columns of a unitary matrix $U \in \mathrm{U}(2)$. The eigenvalues $\left(\lambda_{1}, \lambda_{2}\right)$ are the diagonal entries of a diagonal matrix $\Lambda$. Due to the gap condition, we require $\lambda_{1} \neq \lambda_{2}$, therefore, $\Lambda \in$ $\operatorname{Conf}_{2}(\mathbb{R}):=\left\{\left(\lambda_{1}, \lambda_{2}\right) \in \mathbb{R}^{2}: \lambda_{1} \neq \lambda_{2}\right\}$ [the notation $\left(\lambda_{1}, \lambda_{2}\right)$ refers to a pair of ordered points along the real line]. The eigenvalue decomposition of $\mathcal{H}$ is $\mathcal{H}=U \Lambda U^{-1}$. Thus, our parametrization begins with a map $p: \mathrm{U}(2) \times \operatorname{Conf}_{2}(\mathbb{R}) \rightarrow X$ which is defined by sending a pair $(U, \Lambda) \in \mathrm{U}(2) \times \operatorname{Conf}_{2}(\mathbb{R})$ to the Hamiltonian $\mathcal{H}=U \Lambda U^{-1}$.

The map $p$ is the starting point for our parametrization, but there are two forms of redundancy which we must account for before we have a one-to-one parametrization of $X$. First, the eigenvectors are only defined up to multiplicaton by a unit complex scalar (the gauge invariance). This defines an action of the group $\mathrm{U}(1) \times \mathrm{U}(1)$ on $\mathrm{U}(2)$, namely, multiplying $U$ on the right by a diagonal unitary matrix. Because $\mathcal{H}$ is invariant under this group action, we can replace $\mathrm{U}(2)$ with the quotient group $\mathrm{U}(2) / \mathrm{U}(1) \times \mathrm{U}(1)$. Second, the ordering of the eigenvalues and eigenvectors is not uniquely determined, as long as they are reordered simultaneously. To be precise, if $\sigma$ is the $2 \times 2$ matrix representing the swap permutation, it is easy to verify that $(U, \Lambda) \mapsto\left(U \sigma, \sigma^{-1} \Lambda \sigma\right)$ leaves $\mathcal{H}$ invariant. This defines an action of the group $\mathbb{Z}_{2}$ which we must also divide out. By removing these two redundancies, the parametrization of a given Hamiltonian is uniquely defined, so we have the description of $X$ as

$$
X=\left(\frac{\mathrm{U}(2)}{\mathrm{U}(1) \times \mathrm{U}(1)} \times \operatorname{Conf}_{2}(\mathbb{R})\right) / \mathbb{Z}_{2} .
$$

The equals sign here denotes a homeomorphism of topological spaces. Both of the factor spaces are, like $X$, defined by systems of equations, but they are much more familiar in topology. The space $\mathrm{U}(2) / \mathrm{U}(1) \times \mathrm{U}(1)$ is a classical example

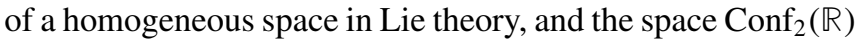
arises in connection with the braid group; both play an important role in algebraic topology in the context of classifying spaces $[70,77,78]$.

The characterization of $X$ we developed is already sufficient for many purposes, but we can simplify it further. As a first simplification, we recognize the space $\mathrm{U}(2) / \mathrm{U}(1) \times \mathrm{U}(1)$ as the Bloch sphere $\mathbb{C} P^{1}=S^{2}$. Another

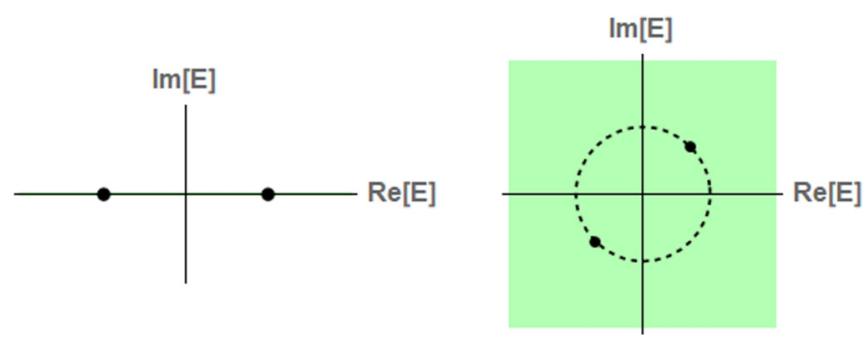

FIG. 1. The space of eigenvalues in the Hermitian vs nonHermitian case; $\operatorname{Conf}_{2}(\mathbb{R}) \sim \mathbb{Z}_{2}$ while $\operatorname{Conf}_{2}(\mathbb{C}) \sim S^{1}$.

simplification we can make (cf. Fig. 1) is to deform the space $\operatorname{Conf}_{2}(\mathbb{R})=\left\{\left(\lambda_{1}, \lambda_{2}\right) \in \mathbb{R}^{2}: \lambda_{1} \neq \lambda_{2}\right\}$ into the discrete space $\mathbb{Z}_{2}=\{+1,-1\}$ (a form of spectral flattening). Because we can choose the deformation (indicated by the symbol $\sim$ ) in a way that respects the $\mathbb{Z}_{2}$ group action, we can retain the parametrization throughout the deformation. We therefore conclude that

$$
\begin{aligned}
X & \sim\left(S^{2} \times \mathbb{Z}_{2}\right) / \mathbb{Z}_{2} \\
& =S^{2} .
\end{aligned}
$$

The intuitive interpretation is that the $S^{2}$ represents one (e.g., the lower-energy) eigenvector of the Hamiltonian $\mathcal{H}$ on the Bloch sphere.

\section{Computing the topological classification}

Now that we understand the target space $X$, we are ready to solve the topological classification problem. As shown in Fig. 2, the torus can be thought of as a rectangle with opposite sides identified. The boundary of this rectangle is called the one-skeleton of the torus, and it contains information about 1D invariants, while the interior of the rectangle is known as the two-cell and contains information about $2 \mathrm{D}$ invariants.

The general strategy is as follows. First, we compute the homotopy groups $\pi_{n}(X)$, which describe topological invariants of various dimensions; this is a preliminary step which provides data we need to compute $\left[T^{2}, X\right]$. The homotopy groups $\pi_{n}(X)$ are defined in terms of maps on the $n$-sphere rather than on the torus; we will be interested in $S^{1}$ and $S^{2}$ since the torus $T^{2}$ has nontrivial cycles in 1D and 2D. Next, we use these data to compute $\left[T_{1}^{2}, X\right]$, where $T_{1}^{2}$ is the one-skeleton of the torus. Finally, we study extensions to the two-cell of the torus; this is the key step. Notationally, if $f \in\left[T_{1}^{2}, X\right]$ is a homotopy class of maps on the one-skeleton, we write $\left[T^{2}, X\right]^{f}$ to denote the set of homotopy classes of extensions of $f$ to the two-cell, i.e., maps in $\left[T^{2}, X\right]$ which restrict to $f$ on the one-skeleton. As a technicality which we elaborate on in Sec. II D, we begin by studying pointed homotopy sets, denoted $\left[T^{2}, X\right]_{*}^{f}$ (so that for now, all maps and homotopies preserve base points).

Following our outlined strategy, we begin by computing the homotopy groups $\pi_{n}(X)=\left[S^{n}, X\right]_{*}$ (considering pointed homotopies). For $X=S^{2}$, the first few are well-known [70]:

$$
\begin{aligned}
& \pi_{1}(X)=0, \\
& \pi_{2}(X)=\mathbb{Z},
\end{aligned}
$$




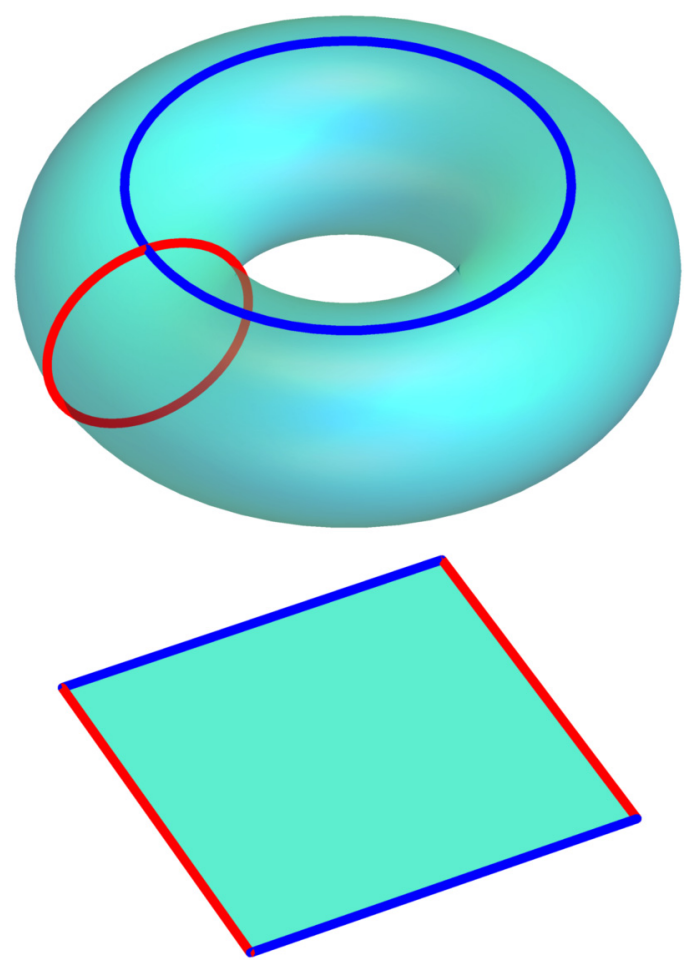

FIG. 2. The torus can be constructed out of a rectangle by gluing together the two red lines and the two blue lines. These lines become closed loops on the torus. Taken together, these two loops form the one-skeleton of the torus, while the cyan interior of the rectangle forms the two-cell of the torus. The one-skeleton $T_{1}^{2}=S^{1} \vee S^{1}$ consists of two circles joined at a common base point, i.e., the "bouquet" of two circles; we refer to these circles as $a$ and $b$ following the mathematical literature.

$$
\pi_{3}(X)=\mathbb{Z}
$$

These correspond physically to topological invariants in various dimensions. We understand $\pi_{1}(X)=0$ as a statement of the fact that there are no one-dimensional topological invariants in Hermitian systems (without additional symmetry protection). On the other hand, $\pi_{2}(X)=\mathbb{Z}$ is a statement about $2 \mathrm{D}$ topological insulators. The fact that this integer invariant is given by the Chern number is slightly subtle, but can be understood, e.g., in terms of the Chern-Weil theory [79]. The three-dimensional "Hopf" invariant is unstable, meaning it does not survive in the presence of additional bands. Nonetheless, it is still of interest in recent works [80-83].

The classification of maps on the one-skeleton $\left[T_{1}^{2}, X\right]_{*}$ is entirely straightforward since $T_{1}^{2}=S^{1} \vee S^{1}$ is the wedge product ("bouquet") of two circles, i.e., two circles joined at a common base point (see Fig. 2). Therefore, $\left[T_{1}^{2}, X\right]_{*}=$ $\pi_{1}(X) \times \pi_{1}(X)=\pi_{1}(X)^{2}$. But, we found that $\pi_{1}(X)=0$, so

$$
\left[T_{1}^{2}, X\right]_{*}=0 \text {. }
$$

The upshot of the result that $\left[T_{1}^{2}, X\right]_{*}=0$ is that we can assume our Hamiltonian $H$ is constant on the one-skeleton of the torus (by continuously deforming it). In other words, we can identify the one-skeleton of the torus to a single point. But then we obtain a sphere, so in this case the extension problem is trivial, and we have

$$
\left[T^{2}, X\right]_{*}^{0}=\pi_{2}(X)=\mathbb{Z},
$$

where $0: T_{1}^{2} \rightarrow X$ denotes the constant map. We have the result

$$
\left[T^{2}, X\right]_{*}=\mathbb{Z}
$$

which constitutes the solution to the classification problem in the Hermitian case.

\section{Action of $\pi_{1}(X)$ on $\pi_{2}(X)$}

Although the obtained classification is complete, there are some important issues regarding base points which we have not yet discussed. More precisely, we have computed the pointed homotopy set $\left[T^{2}, X\right]_{*}$ rather than the free homotopy set $\left[T^{2}, X\right]$. However, in the considered physical setting there is no reason to prefer a particular base point, thus it would be interesting to study how the homotopy class might change as the base point changes; in other words, we are really interested in the free homotopy. In this section, we explain why the two sets could in principle be different, and why the difference can be described in terms of an action of $\pi_{1}(X)$ on $\pi_{2}(X)$. Furthermore, although the extension problem was trivial in the Hermitian case, it will not be in the non-Hermitian case, so this is a good time to point out the features which are absent in the Hermitian case which make the non-Hermitian setting richer. The action of $\pi_{1}(X)$ on $\pi_{2}(X)$ will also play a central role in understanding the general features of this extension problem.

As a central example, we first describe the nontrivial action of $\pi_{1}(X)$ on $\pi_{2}(X)$ for $X=\mathbb{R} P^{2}=S^{2} / \mathbb{Z}_{2}$ (the sphere with antipodal points identified), which will provide the intuition for the non-Hermitian case. We represent elements of $\mathbb{R} P^{2}$ interchangeably as lines or as ellipsoids with a single axis of rotational symmetry, either of which can be thought of as unit vectors with opposite directions identified (due to the symmetry the objects possess). We think of an element of $\pi_{2}\left(R P^{2}\right)$ as a texture of ellipsoids on the sphere. As an example, a fixed "skyrmion" texture, which is the generator of $\pi_{2}\left(\mathbb{R} P^{2}\right)=\mathbb{Z}$, can be visualized by attaching to $x \in S^{2}$ the ellipsoid aligned with the vector $x$.

We now define a continuous deformation of an arbitrary $\mathbb{R} P^{2}$ texture by continuously rotating each ellipsoid in place up to $\pi$ radians around the $y$ axis. This continuous deformation defines a homotopy $H: S^{2} \times[0,1] \rightarrow \mathbb{R} P^{2}$ with $H(\cdot, t) \in$ $\pi_{2}\left(\mathbb{R} P^{2}\right)$ for $t \in[0,1]$ (but not necessarily preserving base points the whole time). The map $H(\cdot, 0) \mapsto H(\cdot, 1)$ thus defines an automorphism of $\pi_{2}\left(\mathbb{R} P^{2}\right)$ which is specifically realized via a homotopy on each element. Whether this automorphism is nontrivial depends on the choice of base point of $\mathbb{R} P^{2}$. Indeed, for the skyrmion texture as described above, if we choose the $y$ axis as the base point $* \in \mathbb{R} P^{2}$, then $H$ is a pointed homotopy, and the automorphism of $\pi_{2}\left(\mathbb{R} P^{2}\right)$ is trivial. In this case, when we choose a lift of the base point to $S^{2}$, the loop $H(*, \cdot)$ lifts to the constant path in $S^{2}$. On the other hand, suppose we choose the $z$ axis as the base point $*$ of $\mathbb{R} P^{2}$. Then, $H$ is a free homotopy since $H(*, t)$ for $t \neq 0,1$ is not kept fixed. Accordingly, when we start 
with $H(\cdot, 0)$ representing $1 \in \pi_{2}\left(\mathbb{R} P^{2}\right)$, the class of $H(\cdot, 1)$ is $-1 \in \pi_{2}\left(\mathbb{R} P^{2}\right)$, so the "skyrmion" texture is continuously deformed into its mirror-image "antiskyrmion" texture. Evidently, this could not be done if the target space were $S^{2}$. The homotopy $H$ thus defines a nontrivial automorphism of $\pi_{2}\left(\mathbb{R} P^{2}\right)$. In this case, when we lift the loop $H(*, \cdot)$ to a path in $S^{2}$, the end points of this path will be antipodal points on $S^{2}$.

In the example above, we observe that when the loop $H(*, \cdot)$ lifts to a path in $S^{2}$ whose end points coincide, the automorphism of $\pi_{2}$ is trivial, whereas when the loop lifts to a path that connects two antipodal points, the automorphism is nontrivial. Below, we formalize this observation by defining an action of $\pi_{1}(X)$ on $\pi_{2}(X)$, where $X$ is an arbitrary space. In this action, the automorphism of $\pi_{2}(X)$ coming from the action of a loop $\gamma$ in $X$ can be realized by a free homotopy $H: S^{2} \times[0,1] \rightarrow X$ with $H(*, t)=\gamma(t)$ [note that $\gamma(t)$ is an element of $\pi_{1}(X)$ ]. The behavior of $H$ at the base point determines the relationship between $H(\cdot, 0)$ and $H(\cdot, 1)$ in $\pi_{2}(X)$. Given a loop $\gamma$ in $X$ and a map $f: S^{2} \rightarrow X$, we can produce a homotopy $H: S^{2} \times[0,1] \rightarrow X$ such that $H(p, 0)=f(p)$ and $H(*, t)=\gamma(t)$ using the homotopy extension property [70]. In the example above for the specific case $X=\mathbb{R} P^{2}$, we provide an explicit construction of such a homotopy by producing a continuous family of homeomorphisms (namely rotations) of $X$ restricting to $\gamma$ on the base point, and using this to define $H$ at every point on $S^{2}$.

To more rigorously define the action of $\pi_{1}(X)$ on $\pi_{2}(X)$, we use the theory of covering spaces [70]. The main theorem we need concerns the homotopy groups of a covering space $Y$ with covering map $p: Y \rightarrow X$. It states that the map induces an isomorphism on all $\pi_{n}$ for $n>1$, and an injection on $\pi_{1}$. Intuitively, the covering space is "unwrapping" some portion of the $\pi_{1}$ while leaving the rest of the homotopy unchanged. In fact, there is a one-to-one correspondence between connected covering spaces and subgroups $\pi_{1}(Y) \subset \pi_{1}(X)$. The universal covering space $\tilde{X}$ is the covering space corresponding to the trivial subgroup.

There is an action of $\pi_{1}(X)$ on any regular covering space $Y$ [one for which $\pi_{1}(Y) \subset \pi_{1}(X)$ is a normal subgroup] by deck transformations. To define this action, we must specify where a loop $\gamma$ at the base point $x_{0} \in X$ sends a point $y \in Y$. For the base point $y_{0} \in Y$, we can lift the loop $\gamma$ to a path $\tilde{\gamma}$ with $\tilde{\gamma}(0)=y_{0}$, and send $y_{0}$ to $\tilde{\gamma}(1)$. For any other point $y \in Y$ lying over $x \in X$, we must first transport the loop $\gamma$ to a loop $\gamma^{\prime}$ at $x$ by choosing a path from $x_{0}$ to $x$. If we choose a different path, the transported loop is well defined up to conjugation by the loop formed by composing the two chosen paths. As before, we lift $\gamma^{\prime}$ to a path $\tilde{\gamma}^{\prime}$ with $\tilde{\gamma}^{\prime}(0)=y$, and map $y$ to $\tilde{\gamma}^{\prime}(1)$. As long as $\pi_{1}(Y)$ is a normal subgroup of $\pi_{1}(X)$, this definition is independent of the choice of path from $x_{0}$ to $x$ [70]. Note that the subgroup $\pi_{1}(Y)$ acts trivially on $Y$.

We define the action of $\pi_{1}(X)$ on $\pi_{2}(X)$ in terms of the action of $\pi_{1}(X)$ on the universal cover $\tilde{X}$ by deck transformations. An element $\gamma \in \pi_{1}(X)$ acts on $\tilde{X}$ by deck transformations, inducing an automorphism $\gamma_{*}$ of $\pi_{2}(\tilde{X})$ [since $\tilde{X}$ is simply connected, $\pi_{2}(\tilde{X})$ is independent of base point]. Using the isomorphisms in $\pi_{2}$ coming from the covering map $\tilde{X} \rightarrow X$, we get an induced automorphism $\gamma_{*}$ of $\pi_{2}(X)$. The action of $\pi_{1}(X)$ on $\pi_{2}(X)$ is thus defined as a map

$$
\rho: \pi_{1}(X) \rightarrow \operatorname{Aut}\left(\pi_{2}(X)\right) .
$$

Now we can see the role of base points in defining $\left[T^{2}, X\right]$. We found $\left[T^{2}, X\right]_{*}=\pi_{2}(X)=\mathbb{Z}$ if one allows only pointed homotopies. The only difference if one allows free homotopies is that a free homotopy could incorporate a nontrivial action of $\pi_{1}(X)$ on $\pi_{2}(X)$. Thus, $\left[T^{2}, X\right]$ is the set of orbits of $\left[T^{2}, X\right]_{*}$ under the action of $\pi_{1}(X)$. In the Hermitian case $X=S^{2}, \pi_{1}(X)=0$, so we have

$$
\begin{aligned}
& \rho=0, \\
& {\left[T^{2}, X\right]=\mathbb{Z} .}
\end{aligned}
$$

In the nontrivial case $X=\mathbb{R} P^{2}$, we note that $S^{2} \rightarrow \mathbb{R} P^{2}$ is a double cover, and $\pi_{1}\left(\mathbb{R} P^{2}\right)=\mathbb{Z}_{2}$ acts on $S^{2}$ via the antipodal map (the deck transformation in this setting) and thus on $\pi_{2}\left(S^{2}\right)$ by parity-conditioned negation. The map $\rho$ : $\pi_{1}(X) \rightarrow \operatorname{Aut}\left(\pi_{2}(X)\right)$ thus sends $1 \in \pi_{1}(X)=\mathbb{Z}_{2}=\{0,1\}$ to the automorphism of $\pi_{2}(X)=\mathbb{Z}$ given by multiplication by -1 ; we can identify $\operatorname{Aut}\left(\pi_{2}(X)\right)=\mathbb{Z}_{2}=\{1,-1\}$ (as the two automorphisms of $\mathbb{Z}$ are given by multiplication by \pm 1 ) and write

$$
\rho(a)=(-1)^{a} .
$$

We study $\left[T^{2}, \mathbb{R} P^{2}\right]$ in Sec. III.

\section{CLASSIFICATION OF TWO-BAND NON-HERMITIAN SYSTEMS}

Now that we have seen the structure of the argument applied to the more familiar Hermitian systems, we can see precisely what changes when one adapts it to non-Hermitian systems. Instead of the space of Hermitian matrices $\mathrm{Herm}_{2}(\mathbb{C})$, we start by considering the space of all $2 \times 2$ matrices $\mathrm{M}_{2}(\mathbb{C})$. In the non-Hermitian case, several different gap conditions have been considered, leading to differing results $[25,62]$. The condition we consider here is natural from a mathematical perspective and results in a classification theory which unifies and extends the existing results.

We define our target space of gapped non-Hermitian Hamiltonians as

$$
X=\left\{\mathcal{H} \in \mathrm{M}_{2}(\mathbb{C}): \operatorname{Disc}(\mathcal{H}) \neq 0\right\},
$$

i.e., the space of $2 \times 2$ matrices with nondegenerate eigenvalues. To make clear the relationship to the point-gap and line-gap classifications that have previously been studied, note that we are considering independently at each wave vector $\boldsymbol{k}$ whether or not the complex eigenvalues of $\mathcal{H}$ coincide as points in the complex plane. This is a weaker constraint than used by the point-gap scheme, which considers Hamiltonians whose spectrum misses a point (such as 0) in the complex plane. It is also very different from the line-gap scheme, which considers Hamiltonians whose spectrum misses a line (such as the imaginary axis) in the complex plane. Under these two schemes, it has been found [62] that non-Hermitian Hamiltonians with a point gap have a $\mathbb{Z}$ invariant in dimension one and none in dimension two, while those with a line gap have a $\mathbb{Z}$ invariant in dimension two and none in dimension one. 
Our gap condition is, however, equivalent to the definition of separable bands from Ref. [39].

\section{A. Characterizing the target space}

With our definition of $X$ in terms of the local gap condition, we need to parametrize $X$ as before in order to compute $\left[T^{2}, X\right]$. The structure of the argument is the same as before; here, we highlight only the relevant differences.

Note that we can again perform an eigendecomposition, just as we did in the Hermitian case. Indeed, a non-Hermitian matrix with nondegenerate eigenvalues is diagonalizable (e.g.. from the theory of the Jordan normal form [84]). We only have two modifications to consider. First, the eigenvectors are no longer orthogonal, so our matrix of eigenvectors is $G \in \mathrm{GL}_{2}(\mathbb{C})$ instead of $U \in \mathrm{U}(2)$. Second, the eigenvalues can be complex, so the space $\operatorname{Conf}_{2}(\mathbb{R})$ is replaced with the space $\operatorname{Conf}_{2}(\mathbb{C})$, i.e., the configuration space of ordered pairs of distinct points in the complex plane.

In the Hermitian case, we have argued that the description has a $\mathrm{U}(1) \times \mathrm{U}(1)$ redundancy as well as a $\mathbb{Z}_{2}$ redundancy. In the non-Hermitian case, the $\mathrm{U}(1) \times \mathrm{U}(1)$ redundancy in the definition of the eigenvectors becomes a $\mathrm{GL}_{1}(\mathbb{C}) \times \mathrm{GL}_{1}(\mathbb{C})$ redundancy $\left[\right.$ recall $\mathrm{GL}_{1}(\mathbb{C})=\mathbb{C}^{\times}$, i.e., the complex plane without the origin]. The $\mathbb{Z}_{2}$ redundancy remains unchanged. Therefore, we have a first description of our target space

$$
X=\left(\frac{\mathrm{GL}_{2}(\mathbb{C})}{\mathrm{GL}_{1}(\mathbb{C}) \times \mathrm{GL}_{1}(\mathbb{C})} \times \operatorname{Conf}_{2}(\mathbb{C})\right) / \mathbb{Z}_{2}
$$

As before, while this expression is sufficient for many calculations, we can simplify it to make a clearer picture of the novelties in the non-Hermitian setting. The first factor $\mathrm{GL}_{2}(\mathbb{C}) / \mathrm{GL}_{1}(\mathbb{C}) \times \mathrm{GL}_{1}(\mathbb{C})$, corresponding to the eigenvectors, is homotopy equivalent to $\mathrm{U}(2) / \mathrm{U}(1) \times \mathrm{U}(1)=S^{2}$. This is expected because of the well-known Gram-Schmidt procedure which deforms $\mathrm{GL}_{2}(\mathbb{C})$ into $\mathrm{U}(2)$, but the actual proof is more complicated (see Ref. [85]). The second factor, $\operatorname{Conf}_{2}(\mathbb{C})$, is evidently more interesting than the $\operatorname{Conf}_{2}(\mathbb{R})$ encountered in the Hermitian case. As we see in Fig. 1, Conf $_{2}(\mathbb{C}) \sim S^{1}$, where a single loop around $S^{1}$ corresponds to the pair of eigenvalues winding around each other once before returning to their original positions (with the same ordering). Thus, we can already see the combination of oneand two-dimensional structure in our characterization

$$
X=\left(S^{2} \times S^{1}\right) / \mathbb{Z}_{2}
$$

This is a key result and will guide our understanding in later sections.

\section{B. Computing the topological classification}

Now that we have a simple characterization of the space $X$, we can begin to study the topological classification. The homotopy groups are easily obtained from the description $X=\left(S^{2} \times S^{1}\right) / \mathbb{Z}_{2}$. Indeed, $X$ has a double cover $Y=S^{2} \times S^{1}$ which has $\pi_{1}(Y)=\mathbb{Z}$ and $\pi_{2}(Y)=\mathbb{Z}$. From general properties of double covers (Sec. IID), the covering map $Y \rightarrow X$ induces an isomorphism in $\pi_{2}$ and "unwraps" $\pi_{1}$ to some extent. To be precise, $\pi_{1}(Y)=\mathbb{Z} \subset \pi_{1}(X)$, and the quotient is $\pi_{1}(X) / \pi_{1}(Y)=\mathbb{Z}_{2}$. This is consistent with $\pi_{1}(X)=\mathbb{Z}$, which we verify in Appendix [and $\pi_{1}(Y)$ sits inside as the even integers]. To gain some insight here, we consider the projections $\Pi_{2}: X \rightarrow S^{2} / \mathbb{Z}_{2}=\mathbb{R} P^{2}$ and $\Pi_{1}: X \rightarrow S^{1} / \mathbb{Z}_{2}=$ $S^{1}$. The map $\Pi_{2}$ selects the unordered eigenvectors and the map $\Pi_{1}$ selects the unordered eigenvalues (after deformations) of $\mathcal{H}$. As we verify in Appendix, $\Pi_{1}$ induces an isomorphism on $\pi_{1}$, so $\pi_{1}(X)=\mathbb{Z}$. On the other hand, $\Pi_{2}$ induces an isomorphism on $\pi_{2}$ and reduction mod two on $\pi_{1}\left[\pi_{1}\left(\mathbb{R} P^{2}\right)=\mathbb{Z}_{2}\right]$. So we have the homotopy groups $\pi_{n}(X)$, namely,

$$
\begin{aligned}
& \pi_{1}(X)=\mathbb{Z}, \\
& \pi_{2}(X)=\mathbb{Z}
\end{aligned}
$$

and all the higher homotopy groups agree with those of the sphere $S^{2}$. We see that all the uniqueness in the non-Hermitian case originates in the eigenvalue winding in 1D. However, as we discuss below, this drastically changes the topological classification in the higher dimensions as well.

The calculation of the homotopy groups also gives some insight into their nature. The space $\operatorname{Conf}_{2}(\mathbb{C}) / \mathbb{Z}_{2}$ is also known as the unordered configuration space $\mathrm{UConf}_{2}(\mathbb{C})$. The one-dimensional invariant is given by the winding of the eigenvalues in $\mathrm{UConf}_{2}(\mathbb{C})$ (so they are allowed to swap after a complete cycle). The two-dimensional invariant of $X$ comes from the unordered eigenvectors as an element of $\mathbb{R} P^{2}$. Moreover, since $S^{2} \rightarrow \mathbb{R} P^{2}$ induces an isomorphism in $\pi_{2}$, we see that any map $S^{2} \rightarrow \mathbb{R} P^{2}$ can be lifted to a map $S^{2} \rightarrow S^{2}$. In other words, for a family of Hamiltonians parametrized by a sphere, one can consistently choose a global ordering of the complex eigenenergies. Then, the two-dimensional $\mathbb{Z}$ invariant is just the ordinary Chern number.

As an additional conceptual simplification, it is convenient to think of $X$ as a "proxy" space which closely resembles $\mathbb{R} P^{2}$. Formally, this is because the map $X \rightarrow \mathbb{R} P^{2}$ induces isomorphisms on $\pi_{m}$ for $m>1$ and is the reduction modulo 2 $\left(\mathbb{Z} \rightarrow \mathbb{Z}_{2}\right.$ ) on $\pi_{1}$, and therefore for our purposes remembers all important homotopy-theoretic data. This analogy facilitates visualization of the following calculation, and leads to particular physical insights outlined in Sec. IV E below.

Now, we are ready to study $\left[T^{2}, X\right]_{*}$. We use the same approach as in the Hermitian case. First, we find for the one-skeleton that

$$
\left[T_{1}^{2}, X\right]_{*}=\mathbb{Z}^{2}
$$

This is an intuitive result since we have a pair of integers describing the eigenvalue winding in each direction on the torus. Now fixing $f: T_{1}^{2} \rightarrow X$, we need to compute $\left[T^{2}, X\right]_{*}^{f}$. We identify $f$ with $(a, b) \in \mathbb{Z}^{2}$ describing its winding in both directions, and write $f=(a, b)$. Recall that in the Hermitian case, because the map $f$ was trivial on the one-skeleton, we could replace extensions with elements of $\pi_{2}(X)$. Here, because $f$ is nontrivial, we need a more sophisticated approach. Our approach is inspired by obstruction theory [78], but we present it in elementary terms.

Our approach is to compute $\left[T^{2}, X\right]_{*}^{f}$ by defining an action of $\pi_{2}(X)$ on $\left[T^{2}, X\right]_{*}^{f}$. For the purpose of these constructions, it is most helpful to visualize elements of $[M, X]$ as $\mathbb{R} P^{2}$ 


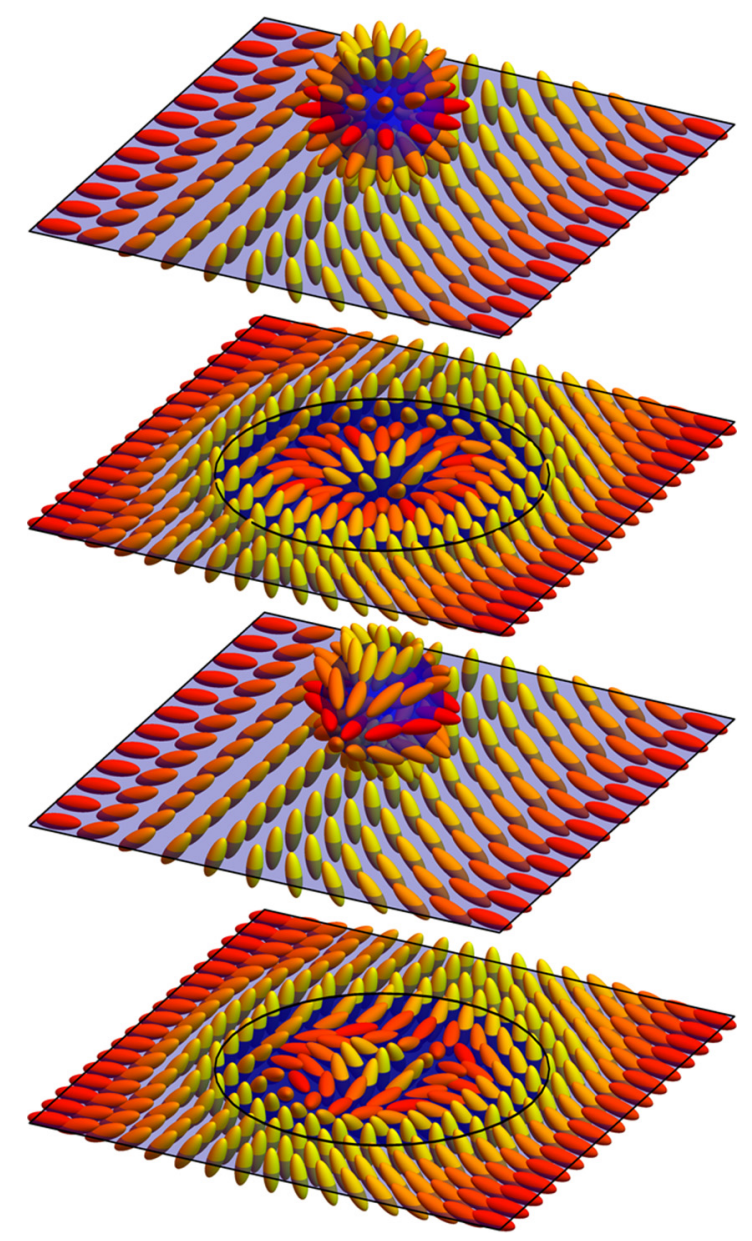

FIG. 3. A texture on the sphere is merged with a texture on the torus to form a new texture on the torus. Two configurations are shown, a skyrmion and an antiskyrmion.

textures on $M$. The action is defined as in Fig. 3 by gluing a small sphere onto the torus. In this figure, we show two configurations, a skyrmion and an antiskyrmion, on $S^{2}$; later, we will discuss how one can continuously go from one to the other, but for now, we just compare the two textures. Starting with a sphere, one can puncture the sphere and the torus at a point, inflate these points, and glue the resulting boundaries together, resulting in a flattened out version of the texture from the sphere now residing on the torus. This gluing process works as long as the ellipsoid on the sphere and the torus at the point of contact have the same orientation. In this way, $\pi_{2}(X)$ acts on $\left[T^{2}, X\right]_{*}^{f}$, modifying any given texture on the torus to produce a new texture on the torus with the same behavior on the one-skeleton. The action can be shown to be transitive; for proofs of these claims using cohomology in the context of obstruction theory, see [78,86,87].

We have a transitive group action of $\pi_{2}(X)$ on $\left[T^{2}, X\right]_{*}^{f}$; by the orbit-stabilizer theorem, $\left[T^{2}, X\right]_{*}^{f}=\pi_{2}(X) / \operatorname{Stab}_{\pi_{2}(X)}(\phi)$ (as sets), where $\operatorname{Stab}_{\pi_{2}(X)}(\phi)$ is the stabilizer of some chosen $\phi \in\left[T^{2}, X\right]_{*}^{f}$ [84]. The stabilizer consists of textures on the sphere which are not homotopic but which become homotopic once glued onto the torus. It turns out that the only way this can occur arises from the action $\rho: \pi_{1}(X) \rightarrow \operatorname{Aut}\left(\pi_{2}(X)\right)$
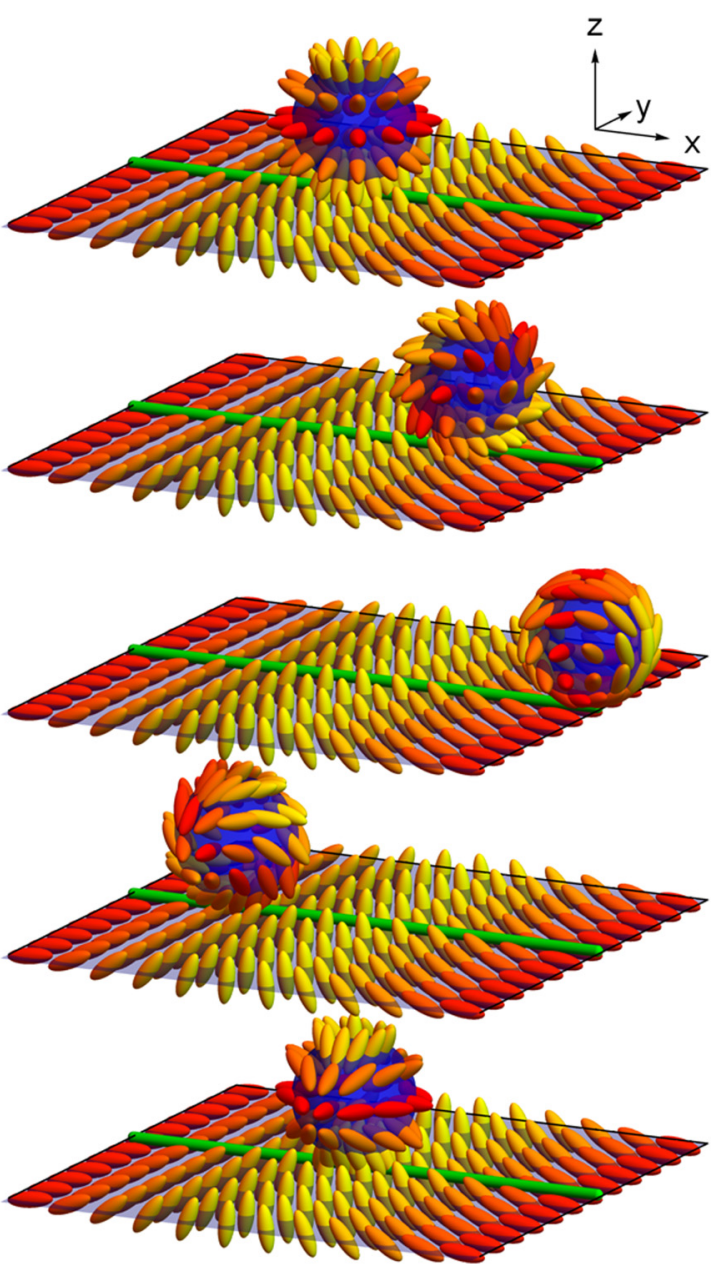

FIG. 4. An $\mathbb{R} P^{2}$ texture on a sphere and a torus, drawn as a field of ellipsoids; the figure illustrates the mechanism by which the action of $\pi_{1}(X)$ on $\pi_{2}(X)$ leads to a reduction from a $\mathbb{Z}$ invariant to a $\mathbb{Z}_{2}$ invariant on the torus. The texture on the torus has nontrivial winding in the $x$ direction, while the texture on the sphere has nontrivial "Chern number." The sphere is moved around a complete cycle in the $x$ direction, and meanwhile each ellipsoid undergoes a $\pi$ rotation around the $y$ axis. In the end, the texture on the sphere is equivalent to the texture on the $x z$ mirror of the original sphere. The color indicates the angle of rotation around the $y$ axis.

[87]. In Fig. 4, we see the mechanism by which this occurs: a sphere with a skyrmion texture can be moved around a nontrivial cycle on the torus such that it ends up with an antiskyrmion texture. If two spheres with skyrmion textures are glued onto the torus and only one is moved around, then we are left with a skyrmion and an antiskyrmion, which can annihilate. Thus, $1+1=2 \in \pi_{2}(X)$ generates $\operatorname{Stab}_{\pi_{2}(X)}(\phi)$, as once glued to the torus, $1+1$ can become $1+(-1)=0$. One way to understand this is that although $\pi_{2}(X)$ is defined in terms of pointed homotopies, a pointed homotopy on the torus can be realized which results in a free homotopy on glued spheres [making it impossible to consistently choose a lift in $\pi_{2}\left(S^{2}\right)$ ]. However, not all free homotopies can be realized by moving the sphere around on the torus. The only ones which can be realized are those coming from $\left[T_{1}^{2}, X\right]_{*}$ [via the action of $\pi_{1}(X)$ on $\pi_{2}(X)$ ]. Generalizing the observation 
that $1=-1$ in the quotient and thus $1-(-1) \in \operatorname{Stab}_{\pi_{2}(X)}(\phi)$, we see that $\operatorname{Stab}_{\pi_{2}(X)}(\phi)$ is generated by elements of the form $s-\rho(f(\gamma)) s$, where $s \in \pi_{2}(X)$ and $\gamma \in \pi_{1}\left(T^{2}\right)$. In other words,

$$
\left[T^{2}, X\right]_{*}^{(a, b)}=\pi_{2}(X) /\langle 1-\rho(a), 1-\rho(b)\rangle .
$$

The angle brackets denote the subgroup generated by a collection of elements; the statement is that the elements of $\pi_{2}(X)$ which become trivial on $T^{2}$ are precisely those which can be written as linear combinations of the two elements which are obtained by comparing a texture with that obtained by moving it around either the $a$ or $b$ direction.

We know from the preceding discussion that $\left[T^{2}, X\right]_{*}^{f}=$ $\pi_{2}(X) /\langle 1-\rho(a), 1-\rho(b)\rangle$, but we have not yet computed the action of $\pi_{1}(X)$ on $\pi_{2}(X)$. Fortunately, this is straightforward from our description $X=\left(S^{2} \times S^{1}\right) / \mathbb{Z}_{2}$. The even subgroup of $\pi_{1}(X)$ corresponding to the double cover $S^{2} \times S^{1}$ clearly acts trivially on $\pi_{2}(X)$. The odd subgroup acts via deck transformations on $S^{2} \times S^{1}$, which restrict to the antipodal map on $S^{2}$. Because this map is orientation reversing, we see that odd elements of $\pi_{1}(X)$ act by negation on $\pi_{2}(X)$. Compare this with Fig. 4, where we illustrate this claim for $\mathbb{R} P^{2}$. Altogether, we find that $\pi_{1}(X)$ acts on $\pi_{2}(X)$ by parityconditioned negation [65]:

$$
\rho(a)=(-1)^{a} .
$$

We observe that $1-(-1)^{a}$ is 0 for $a$ even and 2 for $a$ odd. There are four cases for the parity of $a$ and $b$ to consider; in each case, the stabilizer subgroup in $\pi_{2}(X)=\mathbb{Z}$ is either 0 or $2 \mathbb{Z}$. Therefore,

$$
\left[T^{2}, X\right]_{*}^{(a, b)}=\left\{\begin{array}{l}
\mathbb{Z} \text { if } a, b \text { are both even, } \\
\mathbb{Z}_{2} \text { otherwise. }
\end{array}\right.
$$

This concludes the calculation of the topological classification in the non-Hermitian setting. To summarize, we have

$$
\left[T^{2}, X\right]_{*}=\bigcup_{(a, b) \in \mathbb{Z} \times \mathbb{Z}}\left\{\begin{array}{l}
\mathbb{Z} \text { if } a, b \text { are both even, } \\
\mathbb{Z}_{2} \text { otherwise. }
\end{array}\right.
$$

This should be compared with

$$
\left[T^{2}, \mathbb{R} P^{2}\right]_{*}=\bigcup_{(a, b) \in \mathbb{Z}_{2} \times \mathbb{Z}_{2}}\left\{\begin{array}{l}
\mathbb{Z} \text { if } a, b \text { are both zero, } \\
\mathbb{Z}_{2} \text { otherwise }
\end{array}\right.
$$

obtained for the "proxy" simpler space visualized in Figs. 3 and 4. Finally, we have

$$
\left[T^{2}, X\right]=\bigcup_{(a, b) \in \mathbb{Z} \times \mathbb{Z}}\left\{\begin{array}{l}
\mathbb{N} \text { if } a, b \text { are both even, } \\
\mathbb{Z}_{2} \text { otherwise, }
\end{array}\right.
$$

where $\mathbb{N}$ is the set of natural numbers. We can compare this with

$$
\left[T^{2}, \mathbb{R} P^{2}\right]=\bigcup_{(a, b) \in \mathbb{Z}_{2} \times \mathbb{Z}_{2}}\left\{\begin{array}{l}
\mathbb{N} \text { if } a, b \text { are both zero, } \\
\mathbb{Z}_{2} \text { otherwise. }
\end{array}\right.
$$

The $\mathbb{Z}_{2}$ invariants in Eq. (24) can be understood in terms of the gluing procedure of Fig. 3, which creates a localized positive charge on one band and a localized negative charge on the other. The $\mathbb{Z}_{2}$ nature of the invariant can be understood in terms of the procedure of Fig. 4, which inverts the localized skyrmion texture, i.e., swaps the positive and negative charges
TABLE I. A process demonstrating that the $\mathbb{Z}_{2}$ invariant is unstable under the addition of trivial bands. The total charge across all three bands satisfies a local triviality condition (summing to zero) throughout the process; we ensure this by handling the charges in pairs (one positive and one negative). Stage 1 describes a three-band system where the first two bands have a nontrivial (odd) winding and a nontrivial $\mathbb{Z}_{2}$ invariant. The notation indicates a localized positive charge on band 1 and negative charge on band 2 . This configuration is equivalent to that shown in stage 2 , where we have created a superposed positive and negative charge on band 3 and then separated the four charges into two pairs, one between band 1 and band 3 and one between band 2 and band 3. We apply the odd winding to the first pair to get to stage 3 , and then annihilate the two pairs of opposite chirality to get the trivial stage 4 configuration.

\begin{tabular}{lcccc}
\hline \hline & Stage 1 & Stage 2 & Stage 3 & Stage 4 \\
\hline Band 1 & + & + & & \\
Band 2 & - & - & +- & \\
Band 3 & & -+ & -+ & \\
\hline \hline
\end{tabular}

between the two bands. A consequence of this is that one can start with a pair of skyrmions (total charge 2), move one around the torus in a direction of odd winding to obtain a skyrmion and an antiskyrmion, and then annihilate these to obtain a trivial configuration.

Additionally, the invariants are evidently related to Chern numbers. However, the $\mathbb{Z}_{2}$ case is somewhat subtle because Chern number is not defined when a global ordering of the bands is absent. One could attempt to integrate over the double cover of the torus to remedy this, but the result of such integration is always zero because the contributions from the two sheets cancel each other. Finally, one could simply just integrate over the single torus, ignoring the discontinuity at the boundary. However, such a procedure does not produce a quantized result, and hence such integration does not represent a topological invariant. We discuss the proper way to compute the $\mathbb{Z}_{2}$ invariant later in Sec. IVE, where we also apply the method to study a simple toy Hamiltonian.

In contrast with topological classifications based on $K$ theory, our homotopy approach predicts both stable and unstable invariants. Here, an invariant is said to be stable if it survives in the presence of additional topologically trivial bands [69]. The $\mathbb{Z}$ Chern number and $\mathbb{Z}$ winding numbers are known to be stable since they are predicted using $K$ theory [62]. The $\mathbb{Z}_{2}$ invariant we predict using homotopy theory is unstable. This is best seen in an example, so in Table I we describe an explicit procedure by which an additional trivial band can trivialize the $\mathbb{Z}_{2}$ invariant.

\section{PHYSICAL INTERPRETATION OF CLASSIFICATION RESULTS}

In this section, we develop a physical framework for understanding the classification result obtained in Sec. III. First, in Sec. IV A we describe a well-known relationship between gapped systems and gapless systems, namely, how a Chern number can be described in terms of Weyl points. Then, in Sec. IVB, we extend this correspondence to the non-Hermitian setting using Weyl points and 
exceptional rings, and use this correspondence to interpret the $\mathbb{Z}_{2}$ invariants.

To strengthen our intuition about the reduced topological classification on a torus, we discuss in Sec. IV C an analogy with the classification of topological defects and textures in nematic liquids [71-73], where a closely related phenomenon has been known for a long time [71]. Similar phenomena have been studied more recently in the context of the fragile topology of real-symmetric Hamiltonians [68,74], which we briefly review in Sec. IV D.

\section{A. Correspondence betwen Chern number and Weyl points in Hermitian systems}

We revisit in this section certain elementary aspects of band topology in Hermitian systems, before shifting our focus in Sec. IV B to non-Hermitian systems. The prototypical example of a topological invariant in Hermitian band theory is the (first) Chern number, which is an integer number assigned to any 2D closed manifold inside the momentum space [1]. It is defined as the integral of Berry curvature over the manifold, divided by $2 \pi$. Importantly, there is an exact mathematical correspondence which allows us to interpret the Chern number on a given manifold in terms of the Weyl points enclosed inside that manifold. To be more precise, recall that Weyl points are pointlike degeneracies of a pair of bands inside the three-dimensional (3D) $\boldsymbol{k}$ space [64]. Depending on their chiral charge $\chi= \pm 1$, each Weyl point (WP) acts as either a source or a sink of a $2 \pi$ quantum of Berry curvature [88]. Since Berry curvature has vanishing divergence away from band degeneracies, it follows from Stokes' theorem that the integral of the Berry curvature on the boundary $M=\partial \mathcal{D}$ of any region (domain) $\mathcal{D}$ is quantized to integer multiples of $2 \pi$, and the Chern number $c$ is exactly equal to the total charge of the Weyl points in $\mathcal{D}$.

Reformulating the statements mathematically, it follows from the definition of the Chern number

$$
c^{\alpha}=\frac{1}{2 \pi} \oint_{M} \boldsymbol{F}^{\alpha}(\boldsymbol{k}) \cdot d^{2} \boldsymbol{k} \in \mathbb{Z}
$$

that

$$
c^{\alpha}=\sum_{\mathrm{WP}^{\alpha} \in \mathcal{D}} \chi_{\mathrm{WP}}^{\alpha} .
$$

In Eqs. (28), $\boldsymbol{F}^{\alpha}(\boldsymbol{k})=i\left\langle\nabla u_{\boldsymbol{k}}^{\alpha}|\times| \nabla u_{\boldsymbol{k}}^{\alpha}\right\rangle$ is the Berry curvature on energy band $\alpha,\left|u_{k}^{\alpha}\right\rangle$ is the corresponding cell-periodic part of the Bloch wave function, and $\nabla$ is the gradient operator in $\boldsymbol{k}$ space. Note that we have fixed one band (labeled $\alpha$ ), and we consider only the Weyl points formed by this band. Furthermore, as WPs are degeneracies of pairs of bands, it can be shown [88] that

$$
\chi_{\mathrm{WP}}^{\alpha}=-\chi_{\mathrm{WP}}^{\beta} \quad \text { for WP formed by bands } \alpha \text { and } \beta \text {, }
$$

meaning that each WP acts as a sink on one of the two bands, and as a source on the other band. Two Weyl points which are both formed by bands $\alpha$ and $\beta$ can annihilate only if their chirality is opposite.

\section{B. Interpreting the $\mathbb{Z}_{2}$ invariant in terms of braiding of Weyl points around exceptional rings}

The correspondence between topological insulator and band nodes becomes more subtle in a non-Hermitian setting. Although the (first) Chern number has been previously considered in non-Hermitian systems [39,40], as it is meaningful for some 2D closed manifolds, non-Hermitian Bloch Hamiltonians have an additional 1D invariant [39]. This 1D invariant interacts nontrivially with the Chern number in the sense that the Chern number can be reduced from $\mathbb{Z}$ to $\mathbb{Z}_{2}$ depending on the $1 \mathrm{D}$ invariant (Sec. III). As discussed above, the complication stems from the complex-valued band energies, which allow for the permutation of two energy bands along a closed trajectory without forming a band degeneracy on the way. The presence of such a trajectory inside the 2D Brillouin zone makes it impossible to globally assign each band a unique band index, and therefore Eq. (28a) cannot be readily applied to compute the first Chern number. Here, we briefly discuss the reduction mod 2 of the Chern number in terms of a braiding of Weyl points around exceptional rings reported in Ref. [65].

To get an insight into the nature of the non-Hermitian counterpart of the Chern number, we find it useful to consider again the correspondence with Weyl points. In non-Hermitian systems, it has been found that Weyl points generically turn into one-dimensional ringlike degeneracies known as exceptional rings $[27,64]$. These exceptional rings have been found to have nontrivial one-dimensional invariant associated with the winding on a circle threaded by the ring (associated with the point-gap classification scheme) as well as a nontrivial Chern number on a sphere large enough to enclose the entire ring (associated with the line-gap scheme). Inside a torus, a small exceptional ring may be considered effectively as a Weyl point, with the understanding that it will generically have some small but nonzero radius. However, one can consider large exceptional rings which thread through the torus, in either direction (inside or outside).

For simplicity, we consider a two-band model on a torus [see Fig. 5(b)]. We assume that the two bands are nontrivially permuted along the $\phi$ direction of the torus, and that originally there are no band degeneracies inside the torus. The nontrivial band permutation is realized by an exceptional nodal line threaded through the torus. Let us now consider the following process: through a local band inversion, we produce a pair of Weyl points of opposite chirality. By appropriately adjusting the Hamiltonian parameters, we transport one of the Weyl points along the $\phi$ direction, while keeping the other Weyl point fixed. Along the path, the transported Weyl point flips upside down. According to Eq. (28c), the flip implies that the Weyl point has effectively reversed the chirality. As a consequence, the two Weyl points now (locally) carry the same chirality, and are not able to annihilate anymore.

Conversely, any pair of Weyl points can be annihilated in a model that exhibits a nontrivial band twist along some direction of a $3 \mathrm{D}$ region $\mathcal{D}$. If the two Weyl points locally have the same chirality, one can still annihilate them by transporting one of the Weyl points along the nontrivial path. We thus observe that the right-hand side of Eq. (28b) is no longer an integer invariant in a non-Hermitian system if there 
(a)

\begin{tabular}{c|ccc} 
& non-Hermitian 2-band & uniaxial nematic & fragile real Hermitian \\
\hline line degeneracy & exceptional line (EL) & disclination line (DL) & nodal line (NL) \\
$\pi_{1}$ charge & $\mathbb{Z}$ winding & $\mathbb{Z}_{2}$ rotation & $\mathbb{Z}_{2}$ Berry phase \\
\hline point-like degeneracy & Weyl point (EL-ring) & hedgehog (DL-ring) & real Dirac point (NL-ring) \\
$\pi_{2}$ charge & $\mathbb{Z}$ Chern number & $\mathbb{Z}$ skyrmion number & $\mathbb{Z}$ Euler class \\
reduced charge on $T^{2}$ & $\mathbb{Z}_{2}$ Chern parity & $\mathbb{Z}_{2}$ texture & $\mathbb{Z}_{2} 2^{\text {nd }}$ Stiefel-Whitney class
\end{tabular}

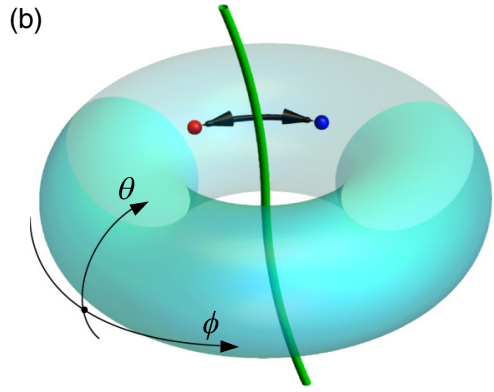

FIG. 5. (a) Comparison of the three physical settings discussed in Secs. IV B, IV C, and IV D. Although the terminology differs, the description of their singularities (topological defects) is very similar. All three systems exhibit topologically protected line and point defects in 3D, mathematically described respectively by $\pi_{1}$ and $\pi_{2}$ of the order-parameter space. In each instance, the 1D and 2D invariants interact nontrivially, meaning that the $\pi_{2}$ charge describing a point node flips sign if it is carried along a closed path with nontrivial $\pi_{1}$ charge. This interaction leads to $\mathbb{Z} \longrightarrow \mathbb{Z}_{2}$ lowering of the topological invariant on the torus, if there is a nontrivial $\pi_{1}$ charge in some direction. (b) A $2 \mathrm{D}$ torus embedded in $3 \mathrm{D}$, with a green curve indicating an exceptional line, and red and blue points indicating Weyl points of opposite chirality. The two angular coordinates on the torus are denoted $\theta$ and $\phi$. Since the $\phi$ direction links with the exceptional line, the torus has nontrivial 1D invariant in the $\phi$ direction. By moving one of the Weyl points around the exceptional line, we can change its chirality and obtain a configuration with total charge 2 from this initial configuration of total charge 0 ; thus, total charge 2 represents a topologically trivial configuration, as we understand in terms of braiding Weyl points around an exceptional line.

is a nontrivial band flip in some direction of the region $\mathcal{D}$. On the other hand, the parity (even vs odd) of the total number of Weyl points inside $\mathcal{D}$ remains invariant, as long as no Weyl points are allowed to move across the boundary $\partial \mathcal{D}$, i.e., as long as $\partial \mathcal{D}$ does not exhibit a gap closing. This change of parity is the manifestation of our $\mathbb{Z}_{2}$ invariant in terms of the correspondence between gapped and gapless systems. For more details on this procedure, see Ref. [65].

Thus, we understand the classification result (24) in terms of Weyl points and exceptional lines and rings inside the torus. While the system is gapped on the torus, it has band degeneracies inside the torus. Exceptional lines and rings which link with the torus are responsible for the 1D part of the classification, i.e., the winding numbers on the oneskeleton; Weyl points are responsible for the 2D part, i.e., the extension to the two-cell. The construction from Sec. III of a group action of $\pi_{2}(X)$ on $\left[T^{2}, X\right]_{*}^{f}$ can be understood as the insertion of a Weyl point into the interior of the solid torus. The reduction $\bmod 2$ of the $\mathbb{Z}$ invariant under conditions of nontrivial winding is understood in terms of parity flip of Weyl points (see also Fig. 4).

\section{Insights from the physics of nematic liquids}

Nematic liquids [73] are the archetypal example of an ordered phase considered in the context of topological defects and textures [72]. This phase of matter is built up from approximately rodlike molecules, which are randomly positioned (resembling a liquid) but with a frozen orientation (resembling a crystalline solid). The order parameter of a liquid crystal is the so-called director, which is an unioriented axis that describes the local orientation of the molecules. The orderparameter space of such "uniaxial" nematics is therefore

$$
X=S^{2} / \mathbb{Z}_{2}=\mathbb{R} P^{2},
$$

where $S^{2}$ represents a unit vector $\boldsymbol{n}$ aligned with the orientation of the molecules, and the quotient identifies $\boldsymbol{n} \sim \boldsymbol{n}$ to produce the "headless" director. This is exactly the "proxy" space considered in detail in Sec. III.

The order-parameter field of a nematic liquid in 3D may exhibit topological defects, which can be explained using homotopy groups. On the one hand, the first homotopy group $\pi_{1}\left(\mathbb{R} P^{2}\right)=\mathbb{Z}_{2}$ describes a nontrivial twist of the order parameter along a closed path $\left(S^{1}\right)$. More precisely, this is a $\pi$ rotation of the director, and the corresponding defect is described as a disclination line [71]. On the other hand, the second homotopy group $\pi_{2}\left(\mathbb{R} P^{2}\right)$ describes a nontrivial texture of the director on a sphere $\left(S^{2}\right)$, which is colloquially called hedgehog.

Naively, by recalling the correspondence between Chern number and Weyl points from Sec. IV A, one might think that nematic textures on a closed surface $\partial \mathcal{D}$ would be characterized by an integer topological invariant that is in correspondence with the number of hedgehogs in $\mathcal{D}$. However, this conclusion is wrong. It has been recognized by Volovik and Mineev [71] that moving a hedgehog around a disclination line flips its integer topological charge. As a consequence, any pair of hedgehog defects can pairwise annihilate if brought together along a nontrivial trajectory. This is very similar to the way Weyl point chirality in non-Hermitian system is reversed when it is moved around an exceptional line. Therefore, one can draw the following analogy between nonHermitian systems and nematic liquids:

$$
\begin{gathered}
\text { exceptional lines } \longleftrightarrow \text { disclination lines, } \\
\text { Weyl points } \longleftrightarrow \text { hedgehogs. }
\end{gathered}
$$

For nematic liquids, it has been found [87] that the interaction between the $1 \mathrm{D}$ and the $2 \mathrm{D}$ invariants reduces the topological classification of textures on torus from $\mathbb{Z}$ to $\mathbb{Z}_{2}$ whenever there is a nontrivial 1D invariant of the director along some direction of the torus. The $\mathbb{Z}_{2}$ invariant is in 
one-to-one correspondence with the parity of the number of hedgehogs inside the torus [cf. Fig. 5(b)].

\section{Fragile topology of real Hermitian models}

Very recently, the observation that topological invariant on a $1 \mathrm{D}$ subspace can reduce the topological classification on a 2D manifold has also been made in the context of topological band theory. More specifically, this phenomenon was reported $[68,74]$ for fragile topological invariants [89] of models with real-symmetric Hamiltonians. Such condition arises either in the presence of $C_{2} \mathcal{T}$ symmetry (composition of $\pi$ rotation with time reversal) or $\mathcal{P} \mathcal{T}$ symetry (composition of spatial inversion with time reversal) $[67,76,90]$.

Let us first summarize the so-called "stable" topology of such real-symmetric Hermitian models, which correspond to nodal class AI of Ref. [91]. The generic band degeneracy of such Hamiltonians in 3D is a nodal line, protected by a $\mathbb{Z}_{2}$-valued (quantized) Berry phase on closed paths $\left(S^{1}\right)$. Furthermore, nodal lines can be folded to produce closed nodal-line rings, which were reported to carry a $\mathbb{Z}_{2}$-valued monopole charge [92] on the enclosing sphere $\left(S^{2}\right)$. This pair of $\mathbb{Z}_{2}$ invariants mathematically correspond to so-called first and second Stiefel-Whitney class [75,79]. By fine tuning the Hamiltonian parameters, nodal-line rings with a monopole charge can be shrunk to a pointlike degeneracy known as "real Dirac point" [93], resembling the way we considered shrinking exceptional nodal-line rings to Weyl points in Sec. IV B.

In systems with a small number of bands, the groups describing the band nodes of real-symmetric Hamiltonians may be enriched. This phenomenon is called fragile topology, and its presence for real-symmetric Hamiltonians has been linked $[89,94,95]$ to the physics of twisted bilayer graphene near the magic angle [96-98]. Especially, when such Hamiltonian exhibits two occupied and an arbitrary (but larger than two) number of unoccupied bands, the monopole charge becomes an integer [91,93] called a Euler class [66,74,75]. It has been reported [68] that the Euler class of a nodal-line ring flips sign when it is carried along a closed path with nontrivial Berry phase. As a consequence, the topological classification of real-symmetric 2D Hamiltonians with two occupied bands reduces from $\mathbb{Z}$ to $\mathbb{Z}_{2}$ whenever there is a nontrivial Berry phase along some direction of the torus [cf. Fig. 5(b)]. The $\mathbb{Z}_{2}$ invariant that remains from the integer Euler class is again the second Stiefel-Whitney class, which we mentioned above in the context of the stable topology. One thus finds the following analogy between the non-Hermitian two-band Hamiltonians and the fragile topology of real-symmetric Hamiltonians:

$$
\begin{aligned}
\text { exceptional lines }(\mathrm{EL}) & \longleftrightarrow \text { nodal lines }(\mathrm{NL}), \\
\text { Weyl points } & \text { real Dirac points }
\end{aligned}
$$

$$
\text { (EL-rings) (NL-rings). }
$$

The comparison between the various systems considered in Secs. IV B, IV C, and IV D is summarized by the table in Fig. 5(a).

\section{E. Wilson-loop spectra interpretation of the $\mathbb{Z}_{2}$ invariant}

Apart from understanding the $\mathbb{Z}_{2}$ topology in terms of Weyl points in 3D (Sec. IV B), it is also desirable to have an intrinsic $2 \mathrm{D}$ algorithm to determine the $\mathbb{Z}_{2}$ invariant derived in Sec. III B in Eq. (23). Here, we describe such an algorithm. Recall that many Hermitian 2D topological invariants, including the Chern number, appear in the Wilson loop eigenvalue flow $[99,100]$, so this is a natural place to look for features of the $\mathbb{Z}_{2}$ invariant, which we understand as a mod 2 reduction of the Chern number. To define the Wilson loop eigenvalue flow, we slice the torus into loops and study the change of Wilson loop eigenvalues along the family of loops.

Of the many possible loop directions we could choose on the 2D torus, only some of them exhibit the familiar and essential gauge invariance; however, we can always choose a loop direction with this property. The condition for gauge invariance is that the eigenvalues not interchange along the loop direction. Indeed, the two eigenvectors have independent gauge (phase) degrees of freedom, and so if they interchange, the eigenvalue is no longer gauge invariant. However, it is always possible to choose such a loop direction with no eigenvalue interchange (even winding number); if both winding numbers are odd, one can choose the diagonal as the loop direction [101]. In the following discussion, we write $k_{1}$ and $k_{2}$ to denote the two directions on the torus, and we assume that the winding along the $k_{1}$ direction is even.

Under this assumption, we consider a family of parallel loops covering the torus, where each loop is directed along the $k_{1}$ direction. For each loop, the energy order is well defined and we can compute Wilson-loop eigenvalues (Berry phases) for both bands. We use biorthogonal left and right eigenvectors to compute the Berry phases, as is standard for non-Hermitian systems [27]. We consider two cases, depending on whether the winding in the $k_{2}$ direction is even or odd.

In the first case, the winding in the $k_{2}$ direction is even, and we expect a $\mathbb{Z}$ invariant which is the usual Chern number. The Chern number is equal to the winding number say of $\phi_{1}$ as one varies $k_{2}$ (which is well defined because $\phi_{1}$ and $\phi_{2}$ do not swap). Because the two eigenvalues $e^{i \phi_{1}}$ and $e^{i \phi_{2}}$ satisfy $\phi_{1}+\phi_{2}=0 \bmod 2 \pi$, an alternative characterization of the Chern number is as the number of times $\phi_{1}$ and $\phi_{2}$ cross, taken with sign, either at $\phi_{1}=\phi_{2}=0$ or at $\phi_{1}=\phi_{2}=\pi$ (the two crossing numbers are equal).

In the second case, the winding in the $k_{2}$ direction is odd, and we expect a $\mathbb{Z}_{2}$ invariant. The odd winding along the $k_{2}$ direction requires the two eigenvalues to have odd total crossing and swap as we vary $k_{2}$ from $-\pi$ to $\pi$. Because the two bands swap, there is no consistent notion of band ordering and therefore no notion of a signed number of crossings of Wilson loop eigenvalues. However, the crossing number at $\phi_{1}=\phi_{2}=0$ or $\phi_{1}=\phi_{2}=\pi$ can still be taken mod 2, without needing to account for sign, and the sum will be odd. On physical grounds, the crossing number mod 2 at $\phi_{1}=\phi_{2}=\pi$ must be our $\mathbb{Z}_{2}$ invariant since a model with a single crossing at $\phi_{1}=\phi_{2}=0$ can be deformed to a model with both $\phi_{1}$ and $\phi_{2}$ constant at 0 , which is clearly trivial.

In Fig. 6, we illustrate the case where we have a $\mathbb{Z}_{2}$ invariant. We first construct a 3D lattice model [65] with Weyl 


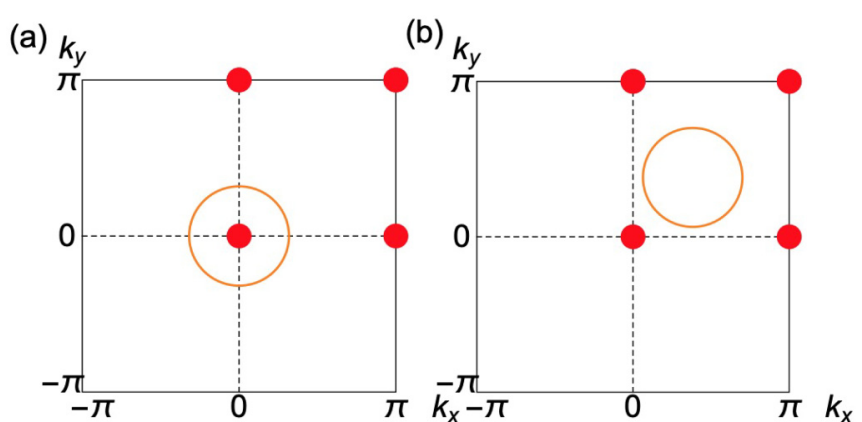

(c)
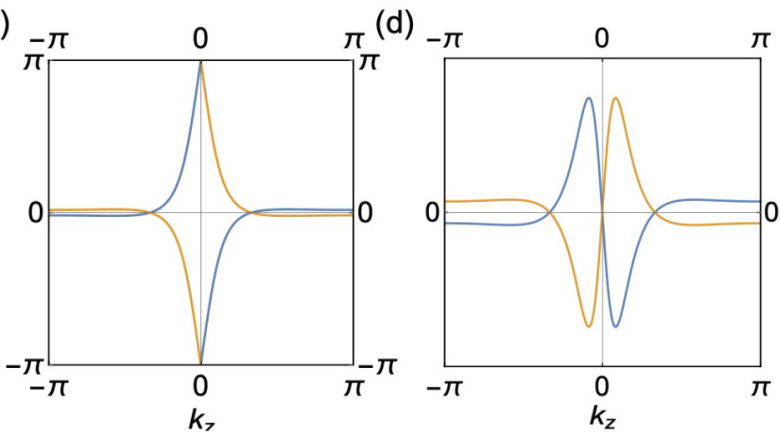

FIG. 6. The Wilson loop eigenvalue flow for the $\mathbb{Z}_{2}$ invariant. (a), (c) Show nontrivial $\mathbb{Z}_{2}$ invariant, while (b) and (d) show trivial $\mathbb{Z}_{2}$ invariant. We construct $2 \mathrm{D}$ models starting with a $3 \mathrm{D}$ model, and restricting to a $2 \mathrm{D}$ surface inside $3 \mathrm{D} k$ space. The $3 \mathrm{D}$ model has four Weyl points (red) located at $k_{z}=0$ plane as shown in (a) and (b). We choose two different cylinders (topologically, toruses) whose projections are shown as orange loops in (a) and (b) for the Wilson loop calculation in (c) and (d). (c) Shows the flow of Wilson loop eigenvalues when the center of the cylinder lies at $\left(k_{x}, k_{y}\right)=(0,0)$. The cylinder encloses one Weyl point and the invariant is nontrivial. We see one crossing (odd) at $\pi$ and two crossings (even) at 0 . (d) Shows the flow of Wilson loop eigenvalues when the center of the cylinder lies at $\left(k_{x}, k_{y}\right)=(1.2,1.2)$. The cylinder encloses no Weyl points and the invariant is trivial. We see zero crossing (even) at $\pi$ and three crossings (odd) at 0.

points and nontrivial winding along one momentum direction:

$$
\begin{aligned}
\mathcal{H}(\boldsymbol{k} ; m)= & e^{i \frac{k_{z}}{2}}\left[\cos \left(\frac{k_{z}}{2}-\frac{\pi}{3}\right) \sin k_{x} \sigma_{x}\right. \\
& +\cos \left(\frac{k_{z}}{2}+\frac{\pi}{3}\right) \sin k_{y} \sigma_{y} \\
& \left.+\left(\sin k_{z} \cos \frac{k_{z}}{2}-2 m \sin \frac{k_{z}}{2}\right) \sigma_{z}\right] .
\end{aligned}
$$

The construction is inspired by the correspondence described in Sec. IV B. For $m>1$, there are four Weyl points at $\left(k_{x}, k_{y}, k_{z}\right)=(0,0,0),(\pi, 0,0),(0, \pi, 0)$, and $(\pi, \pi, 0)$. Here, we take $m=2$ and $k_{r}=1$. Now we can take a cylinder (more precisely a torus) centered at $k_{x}=k_{y}=0$ with certain radius $k_{r}$, small compared to the separation of the Weyl points. This 2D torus embedded in the 3D space of $k_{x}, k_{y}$, and $k_{z}$ will define our 2D model, taking the polar direction on the cylinder to be $k_{1}$ (since there is clearly no winding along this direction) and the $k_{z}$ direction to be $k_{2}$ (since there is odd winding in this direction). For this choice of cylinder, the total parity of the enclosed Weyl points is 1 . On the other hand, if we shift the center of the cylinder away from $k_{x}=k_{y}=0$ to move the Weyl point outside of the cylinder, the total parity of the Weyl points is 0 . We choose our loop direction to be $k_{1}$ (the polar direction on the cylinder) and calculate the Wilson loop eigenvalue flow as we vary $k_{2}\left(k_{z}\right)$. The results for the two choices of cylinder are shown in Fig. 6.

\section{GENERAL CLASSIFICATION OF $N$-BAND MODELS}

We have by now developed a solid understanding of topological invariants in non-Hermitian systems with two bands. Here, we generalize the result to $N$ bands. The structure is even richer, with $\mathbb{Z}$ - and $\mathbb{Z}_{2}$-valued invariants replaced with braid group $\left(\mathbb{B}_{N}\right)$ and cyclic groups $\left(\mathbb{Z}_{k}\right)$ valued invariants. Nevertheless, the overall logic behind the derivation of these results is the same as before.

To start, we use the same gap condition

$$
X=\left\{\mathcal{H} \in \mathrm{M}_{N}(\mathbb{C}): \operatorname{Disc}(\mathcal{H}) \neq 0\right\} .
$$

This means that we only consider "fully gapped" Hamiltonians in which all the complex eigenvalues are distinct. The deformations and redundancies from before generalize directly to this scenario, giving

$$
X=\left(\frac{\mathrm{U}(N)}{\mathrm{U}(1) \times \cdots \times \mathrm{U}(1)} \times \operatorname{Conf}_{N}(\mathbb{C})\right) / \mathbb{S}_{N},
$$

where the unitary quotient space $\mathrm{U}(N) / \mathrm{U}(1) \times \cdots \times \mathrm{U}(1)$ is known as the complex flag manifold, and $\mathbb{S}_{N}$ is the symmetric group, acting via $N \times N$ permutation matrices. In this case, we will not simplify the presentation of $X$ further. Instead, we use well-known results $[70,77,78]$ concerning the homotopy groups of the factors, namely,

$$
\begin{gathered}
\pi_{1}\left(\operatorname{Conf}_{N}(\mathbb{C})\right)=\mathbb{P}_{N}, \\
\pi_{m}\left(\operatorname{Conf}_{N}(\mathbb{C})\right)=0, m>1 \\
\pi_{1}\left(\operatorname{UConf}_{N}(\mathbb{C})\right)=\mathbb{B}_{N}, \\
\pi_{m}\left(\operatorname{UConf}_{N}(\mathbb{C})\right)=0, m>1 \\
\pi_{1}\left(\frac{\mathrm{U}(N)}{\mathrm{U}(1) \times \cdots \times \mathrm{U}(1)}\right)=0, \\
\pi_{2}\left(\frac{\mathrm{U}(N)}{\mathrm{U}(1) \times \cdots \times \mathrm{U}(1)}\right)=\mathbb{Z}^{N-1} .
\end{gathered}
$$

Here, $\mathbb{P}_{N}$ is the pure (ordered) braid group, $\mathbb{B}_{N}$ is the full braid group, and $\operatorname{Conf}_{N}(\mathbb{C})\left[\operatorname{UConf}_{N}(\mathbb{C})\right]$ is the ordered (unordered) configuration space of $N$ points in the complex plane. The first equations are understood in terms of $N$-ordered points in the plane braiding around each other. The last equation describes a Chern number associated to each band, subject to the constraint that the sum over the Chern numbers of all the bands must be zero.

We can compute the homotopy groups of $X$ as before. Since $X$ has a covering space with deck transformation group $\mathbb{S}_{N}$ whose homotopy groups we understand, we obtain immediately $\pi_{2}(X)=\mathbb{Z}^{N-1}$. We use the projection $X \rightarrow$ $\operatorname{Conf}_{N}(\mathbb{C}) / \mathbb{S}_{N}=\operatorname{UConf}_{N}(\mathbb{C})$ with simply connected fiber to 
obtain $\pi_{1}(X)=\mathbb{B}_{N}$. The interpretation of these invariants is a straightforward extension of the two-band case.

Now, we can study $\left[T^{2}, X\right]_{*}$. On the one-skeleton, we have $\left[T_{1}^{2}, X\right]_{*}=\mathbb{B}_{N}^{2}$. Now, $\pi_{1}(X)=\mathbb{B}_{N}$ acts on $\pi_{2}(X)=$ $\mathbb{Z}^{N-1}$ by permutations in the standard representation; this is evident from the $\mathbb{S}_{N}$-covering space and the interpretation of $\pi_{2}(\mathrm{U}(N) / \mathrm{U}(1) \times \cdots \times \mathrm{U}(1))$ as $N$ Chern numbers whose sum is zero.

Let $f: T_{1}^{2} \rightarrow X$ be given by a pair of braids $\left(b_{1}, b_{2}\right)$, and let $\left(\sigma_{1}, \sigma_{2}\right)$ be the corresponding pair of permutations. We will study extensions of $f$ to the two-cell of the torus by computing the stabilizer for the action of $\pi_{2}(X)$ on the set of extensions. The relations on $\pi_{2}(X)=\mathbb{Z}^{N-1}$ are generated by the columns of $\mathbb{1}-\sigma_{1}$ and $\mathbb{1}-\sigma_{2}$ as matrices in the standard matrix representation. As an example, we study the case $\sigma_{1}=(1, \ldots, N)$ is a single $N$ cycle, and $\sigma_{2}=1$ trivial. The notation $\sigma_{1}=(1, \ldots, N)$ means that band 1 goes to band 2, band 2 goes to band 3, etc., and band $N$ goes back to band 1 . We choose a basis $\left\{e_{i}-e_{i+1}\right\}$ for $\mathbb{Z}^{N-1}$. With respect to this basis, we have

$$
1-\sigma_{1}=\left(\begin{array}{ccccccc}
1 & 0 & 0 & \ldots & 0 & 0 & 1 \\
-1 & 1 & 0 & \ldots & 0 & 0 & 1 \\
0 & -1 & 1 & \ldots & 0 & 0 & 1 \\
\vdots & \vdots & \vdots & \ddots & \vdots & \vdots & \vdots \\
0 & 0 & 0 & \ldots & 1 & 0 & 1 \\
0 & 0 & 0 & \ldots & -1 & 1 & 1 \\
0 & 0 & 0 & \ldots & 0 & -1 & 2
\end{array}\right) .
$$

Multiplying on the left by the determinant 1 matrix

$$
\left(\begin{array}{ccccccc}
1 & 0 & 0 & \ldots & 0 & 0 & 0 \\
1 & 1 & 0 & \ldots & 0 & 0 & 0 \\
1 & 1 & 1 & \ldots & 0 & 0 & 0 \\
\vdots & \vdots & \vdots & \ddots & \vdots & \vdots & \vdots \\
1 & 1 & 1 & \ldots & 1 & 0 & 0 \\
1 & 1 & 1 & \ldots & 1 & 1 & 0 \\
1 & 1 & 1 & \ldots & 1 & 1 & 1
\end{array}\right)
$$

we obtain

$$
\left(\begin{array}{ccccccc}
1 & 0 & 0 & \ldots & 0 & 0 & 1 \\
0 & 1 & 0 & \ldots & 0 & 0 & 2 \\
0 & 0 & 1 & \ldots & 0 & 0 & 3 \\
\vdots & \vdots & \vdots & \ddots & \vdots & \vdots & \vdots \\
0 & 0 & 0 & \ldots & 1 & 0 & N-3 \\
0 & 0 & 0 & \ldots & 0 & 1 & N-2 \\
0 & 0 & 0 & \ldots & 0 & 0 & N
\end{array}\right)
$$

whose column space is an index $N$ sublattice of $\mathbb{Z}^{N-1}$. It follows that instead of $N-1$ Chern numbers, the invariant of these $N$ bands reduces to a single $\mathbb{Z}_{N}$ invariant. The intuition is similar to the two-band case. The row vector $(1, \ldots, 1)$ provides the map $\mathbb{Z}^{N-1} \rightarrow \mathbb{Z}_{N}$, so we can interpret the $\mathbb{Z}_{N}$ invariant as the total number of Weyl points $\sum_{i} e_{i}-e_{i+1} \bmod$ $N$ (see Sec. IV E). The result for general permutations is more complicated, but can be worked out on a case-by-case basis by computing the Smith normal form [102].

\section{CONCLUSION}

We have presented a topological classification scheme for gapped non-Hermitian systems, which generalizes existing schemes and finds different types of topological invariants. In particular, we find 1D invariants with values in braid groups, and $2 \mathrm{D}$ invariants with values in $\mathbb{Z}_{N}$ instead of the expected collection of $N-1$ independent Chern numbers. We provided a detailed pedagogical explanation of how this arises from the mathematical phenomenon of the action of $\pi_{1}$ on $\pi_{2}$. We interpreted these classification results in terms of Weyl points and exceptional rings, and connected them to a previously reported nodal braiding in non-Hermitian systems. We illustrate these results using the familiar physics of nematic liquids, and also describe connections to fragile topology of real Hermitian models. Finally, we describe how these invariants are computed, and we illustrate this in a simple computational model.

As we have explained, models representing any of the reported classes can easily be constructed using Weyl points and exceptional rings. This would allow the creation of lattice models in order to experimentally probe edge-state phenomena associated with these invariants, e.g., in optical lattices or synthetic dimension lattices [103]. Then, one could extend the bulk-edge correspondence to this generalized classification and invariants, providing a clearer understanding of the bulkedge correspondence in non-Hermitian systems.

Note added. Recently, a similar work was brought to our attention (Ref. [104]) which arrives at the same classification result; our paper in addition provides a pedagogical derivation and many intuitive ways to understand the classification result, in addition to an algorithm for computing the invariants.

\section{ACKNOWLEDGMENTS}

We thank A. Tiwari for helpful discussions, and R.-J. Slager and A. Tiwari for providing feedback on an early version of our manuscript. C.C.W. and S.F. were supported by a Vannevar Bush Faculty Fellowship (Grant No. N0001417-1-3030) from the U. S. Department of Defense, and by the National Science Foundation Grant No. CBET-1641069. X.-Q.S. was supported by the U.S. Department of Energy Office of Science, Office of High Energy Physics (Grant No. DE-SC0019380) and in part by the Simons foundation. T.B. was supported by the Gordon and Betty Moore Foundation's EPiQS Initiative, Grant GBMF4302, and by the Ambizione Program of the Swiss National Science Foundation, Grant No. 185806.

\section{APPENDIX: HOMOTOPY GROUPS OF $\left(S^{2} \times S^{1}\right) / \mathbb{Z}_{2}$}

In this Appendix, we study the maps $\Pi_{1}$ and $\Pi_{2}$ introduced in Sec. III B and use them to formalize some claims about the homotopy groups of $X=\left(S^{2} \times S^{1}\right) / \mathbb{Z}_{2}$. In particular, we can gain some intuition about the homotopy groups by relating $X$ to $S^{1}$ and to $\mathbb{R} P^{2}$. Furthermore, the covering space structure alone is insufficient to completely determine $\pi_{1}(X)$, whereas these calculations do determine $\pi_{1}(X)$. 
Recall that $\Pi_{1}: X \rightarrow S^{1}$ and $\Pi_{2}: X \rightarrow \mathbb{R} P^{2}$ are the natural projections from $X$. Both $S^{1}$ and $\mathbb{R} P^{2}$ have necessarily forgotten the ordering of the eigenvalues and eigenvectors. Note that once one fixes an ordering on the eigenvectors, an ordering is also determined on the eigenvalues (and vice versa). Thus, the map $\Pi_{2}$ is a fiber bundle with fiber $S^{1}$, and the map $\Pi_{1}$ is a fiber bundle with fiber $S^{2}$. We can formally write this as

$$
\begin{aligned}
& S^{2} \longrightarrow X \stackrel{\Pi_{1}}{\longrightarrow} S^{1}, \\
& S^{1} \longrightarrow X \stackrel{\Pi_{2}}{\longrightarrow} \mathbb{R} P^{2} .
\end{aligned}
$$

From a general property of fiber bundles, we obtain the long exact sequences [70]

$$
\begin{array}{rr}
\cdots \longrightarrow \pi_{m} \longrightarrow \pi_{m}\left(S^{1}\right) & \left(S^{2} \longrightarrow \pi^{\longrightarrow} \longrightarrow\right. \\
\cdots & \pi_{m-1}\left(S^{2}\right) \longrightarrow \pi_{m}\left(\mathbb{R} P^{2}\right) \\
\longrightarrow & \pi_{m}\left(S^{1}\right) \longrightarrow \pi_{m}(X) \longrightarrow \\
\longrightarrow & \text { (A3) }
\end{array}
$$

It follows from the exactness of the sequence in Eq. (A2) and from $\pi_{1}\left(S^{2}\right)=\mathbb{0}=\pi_{0}\left(S^{2}\right)$ that $\pi_{m}(X) \rightarrow \pi_{m}\left(S^{1}\right)$ is an isomorphism for $m=1$, therefore, the one-dimensional invariant is given by the winding of the eigenvalues in $\operatorname{UConf}_{2}(\mathbb{C})$. Furthermore, we find using the exact sequence in Eq. (A3) and using $\pi_{2,3, \ldots}\left(S^{1}\right)=\mathbb{0}$ that $\pi_{m}(X) \rightarrow \pi_{m}\left(\mathbb{R} P^{2}\right)$ is an isomorphism for $m>2$. For $m=2$, this map is still an isomorphism because the map $\pi_{1}\left(S^{1}\right)=\mathbb{Z} \rightarrow \pi_{1}(X)=\mathbb{Z}$ is the inclusion of the even integers. This observation also implies that for $m=1$, the map is a reduction modulo 2, i.e., $\pi_{1}\left(\mathbb{R} P^{2}\right)=$ $\mathbb{Z}_{2}$ remembers the parity of the winding of the unordered eigenvalues.

We remark that a covering space is a special case of a fiber bundle, one whose fiber is discrete. Applying the long exact sequence to $S^{2} \times S^{1} \rightarrow\left(S^{2} \times S^{1}\right) / \mathbb{Z}_{2}$ reproduces the result that the covering map induces an isomorphism in $\pi_{2}$ and an inclusion in $\pi_{1}$, and in fact tells us that $\pi_{1}(X)$ surjects onto $\mathbb{Z}_{2}$ with kernel $\pi_{1}\left(S^{2} \times S^{1}\right)=\mathbb{Z}$. However, one is unable to determine the extension type from this information alone, which is one reason it is beneficial to study the fiber bundles $\Pi_{1}$ and $\Pi_{2}$.
[1] M. Z. Hasan and C. L. Kane, Colloquium: Topological insulators, Rev. Mod. Phys. 82, 3045 (2010).

[2] X.-L. Qi and S.-C. Zhang, Topological insulators and superconductors, Rev. Mod. Phys. 83, 1057 (2011).

[3] D. J. Thouless, M. Kohmoto, M. P. Nightingale, and M. den Nijs, Quantized Hall Conductance in A Two-Dimensional Periodic Potential, Phys. Rev. Lett. 49, 405 (1982).

[4] C. L. Kane and E. J. Mele, $Z_{2}$ Topological Order and the Quantum Spin Hall Effect, Phys. Rev. Lett. 95, 146802 (2005).

[5] J. K. Asbóth, L. Oroszlány, and A. Pályi, A Short Course on Topological Insulators: Band-Structure Topology and Edge States in One and Two Dimensions, Lecture Notes in Physics, Vol. 919 (Springer, 2019).

[6] P. Hořava, Stability of Fermi Surfaces and $K$ Theory, Phys. Rev. Lett. 95, 016405 (2005).

[7] A. Kitaev, Periodic table for topological insulators and superconductors, in Advances in Theoretical Physics: Landau Memorial Conference, edited by V. Lebedev and M. Feigelman, AIP Conf. Proc. No. 1134 (AIP, New York, 2009), p. 22.

[8] S. Ryu, A. P. Schnyder, A. Furusaki, and A. W. W. Ludwig, Topological insulators and superconductors: tenfold way and dimensional hierarchy, New J. Phys. 12, 065010 (2010).

[9] J. Kruthoff, J. de Boer, J. van Wezel, C. L. Kane, and R.J. Slager, Topological Classification of Crystalline Insulators through Band Structure Combinatorics, Phys. Rev. X 7, 041069 (2017).

[10] H. Zhou, C. Peng, Y. Yoon, C. W. Hsu, K. A. Nelson, L. Fu, J. D. Joannopoulos, M. Soljačić, and B. Zhen, Observation of bulk Fermi arc and polarization half charge from paired exceptional points, Science 359, 1009 (2018).

[11] L. Lu, Z. Wang, D. Ye, L. Ran, L. Fu, J. D. Joannopoulos, and M. Soljačić, Experimental observation of Weyl points, Science 349, 622 (2015).

[12] W.-J. Chen, M. Xiao, and C. T. Chan, Photonic crystals possessing multiple Weyl points and the experimental observation of robust surface states, Nat. Commun. 7, 13038 (2016).
[13] J. Noh, S. Huang, D. Leykam, Y. D. Chong, K. P. Chen, and M. C. Rechtsman, Experimental observation of optical Weyl points and Fermi arc-like surface states, Nat. Phys. 13, 611 (2017).

[14] A. Cerjan, S. Huang, M. Wang, K. P. Chen, Y. Chong, and M. C. Rechtsman, Experimental realization of a Weyl exceptional ring, Nat. Photonics 13, 623 (2019).

[15] Q. Wang, M. Xiao, H. Liu, S. Zhu, and C. T. Chan, Optical Interface States Protected by Synthetic Weyl Points, Phys. Rev. X 7, 031032 (2017).

[16] B. Zhen, C. W. Hsu, Y. Igarashi, L. Lu, I. Kaminer, A. Pick, S.L. Chua, J. D. Joannopoulos, and M. Soljačić, Spawning rings of exceptional points out of Dirac cones, Nature (London) 525, 354 (2015).

[17] H. Zhou, J. Y. Lee, S. Liu, and B. Zhen, Exceptional surfaces in $\mathcal{P} \mathcal{T}$-symmetric non-Hermitian photonic systems, Optica $\mathbf{6}$, 190 (2019).

[18] J. M. Zeuner, M. C. Rechtsman, Y. Plotnik, Y. Lumer, S. Nolte, M. S. Rudner, M. Segev, and A. Szameit, Observation of A Topological Transition in the Bulk of a Non-Hermitian System, Phys. Rev. Lett. 115, 040402 (2015).

[19] L. Xiao, X. Zhan, Z. H. Bian, K. K. Wang, X. Zhang, X. P. Wang, J. Li, K. Mochizuki, D. Kim, N. Kawakami, W. Yi, H. Obuse, B. C. Sanders, and P. Xue, Observation of topological edge states in parity-time-symmetric quantum walks, Nat. Phys. 13, 1117 (2017).

[20] W. Hu, H. Wang, P. P. Shum, and Y. D. Chong, Exceptional points in a non-Hermitian topological pump, Phys. Rev. B 95, 184306 (2017).

[21] C. Poli, M. Bellec, U. Kuhl, F. Mortessagne, and H. Schomerus, Selective enhancement of topologically induced interface states in a dielectric resonator chain, Nat. Commun. 6, 6710 (2015).

[22] S. Weimann, M. Kremer, Y. Plotnik, Y. Lumer, S. Nolte, K. G. Makris, M. Segev, M. C. Rechtsman, and A. Szameit, 
Topologically protected bound states in photonic parity-timesymmetric crystals, Nat. Mater. 16, 433 (2016).

[23] X. Zhan, L. Xiao, Z. Bian, K. Wang, X. Qiu, B. C. Sanders, W. Yi, and P. Xue, Detecting Topological Invariants in Nonunitary Discrete-Time Quantum Walks, Phys. Rev. Lett. 119, 130501 (2017).

[24] Y. Choi, S. Kang, S. Lim, W. Kim, J.-R. Kim, J.-H. Lee, and K. An, Quasieigenstate Coalescence in An Atom-Cavity Quantum Composite, Phys. Rev. Lett. 104, 153601 (2010).

[25] D. S. Borgnia, A. J. Kruchkov, and R.-J. Slager, NonHermitian Boundary Modes and Topology, Phys. Rev. Lett. 124, 056802 (2020).

[26] Y. Xu, S.-T. Wang, and L.-M. Duan, Weyl Exceptional Rings in a Three-Dimensional Dissipative Cold Atomic Gas, Phys. Rev. Lett. 118, 045701 (2017).

[27] A. Cerjan, M. Xiao, L. Yuan, and S. Fan, Effects of nonHermitian perturbations on Weyl Hamiltonians with arbitrary topological charges, Phys. Rev. B 97, 075128 (2018).

[28] A. Y. Song, X.-Q. Sun, A. Dutt, M. Minkov, C. Wojcik, H. Wang, I. Williamson, M. Orenstein, and S. Fan, $\mathcal{P} \mathcal{T}$ symmetric topological edge-gain effect, arXiv:1910.10946.

[29] T. Hofmann, T. Helbig, F. Schindler, N. Salgo, M. Brzezińska, M. Greiter, T. Kiessling, D. Wolf, A. Vollhardt, A. Kabaši, C. H. Lee, A. Bilušić, R. Thomale, and T. Neupert, Reciprocal skin effect and its realization in a topolectrical circuit, arXiv:1908.02759.

[30] L. Jin, Topological phases and edge states in a non-Hermitian trimerized optical lattice, Phys. Rev. A 96, 032103 (2017).

[31] X. Ni, D. Smirnova, A. Poddubny, D. Leykam, Y. Chong, and A. B. Khanikaev, $\mathcal{P} \mathcal{T}$ phase transitions of edge states at $\mathcal{P} \mathcal{T}$ symmetric interfaces in non-Hermitian topological insulators, Phys. Rev. B 98, 165129 (2018).

[32] H. Wang, L.-J. Lang, and Y. D. Chong, Non-Hermitian dynamics of slowly varying Hamiltonians, Phys. Rev. A 98, 012119 (2018).

[33] V. Kozii and L. Fu, Non-Hermitian topological theory of finitelifetime quasiparticles: prediction of bulk Fermi arc due to exceptional point, arXiv:1708.05841.

[34] H. Shen and L. Fu, Quantum Oscillation from in-Gap States and a Non-Hermitian Landau Level Problem, Phys. Rev. Lett. 121, 026403 (2018).

[35] M. Papaj, H. Isobe, and L. Fu, Nodal arc of disordered Dirac fermions and non-Hermitian band theory, Phys. Rev. B 99, 201107(R) (2019).

[36] T. Yoshida, R. Peters, and N. Kawakami, Non-Hermitian perspective of the band structure in heavy-fermion systems, Phys. Rev. B 98, 035141 (2018).

[37] P. A. McClarty and J. G. Rau, Non-Hermitian topology of spontaneous magnon decay, Phys. Rev. B 100, 100405(R) (2019).

[38] N. Hatano and D. R. Nelson, Localization Transitions in Non-Hermitian Quantum Mechanics, Phys. Rev. Lett. 77, 570 (1996).

[39] H. Shen, B. Zhen, and L. Fu, Topological Band Theory for Non-Hermitian Hamiltonians, Phys. Rev. Lett. 120, 146402 (2018).

[40] S. Yao, F. Song, and Z. Wang, Non-Hermitian Chern Bands, Phys. Rev. Lett. 121, 136802 (2018).

[41] S. Yao and Z. Wang, Edge States and Topological Invariants of Non-Hermitian Systems, Phys. Rev. Lett. 121, 086803 (2018).
[42] Z. Gong, Y. Ashida, K. Kawabata, K. Takasan, S. Higashikawa, and M. Ueda, Topological Phases of NonHermitian Systems, Phys. Rev. X 8, 031079 (2018).

[43] F. Song, S. Yao, and Z. Wang, Non-Hermitian Topological Invariants in Real Space, Phys. Rev. Lett. 123, 246801 (2019).

[44] H. Wang, J. Ruan, and H. Zhang, Non-Hermitian nodalline semimetals with an anomalous bulk-boundary correspondence, Phys. Rev. B 99, 075130 (2019).

[45] J. C. Budich, J. Carlström, F. K. Kunst, and E. J. Bergholtz, Symmetry-protected nodal phases in non-Hermitian systems, Phys. Rev. B 99, 041406(R) (2019).

[46] Z. Yang and J. Hu, Non-Hermitian Hopf-link exceptional line semimetals, Phys. Rev. B 99, 081102(R) (2019).

[47] J. Carlström and E. J. Bergholtz, Exceptional links and twisted Fermi ribbons in non-Hermitian systems, Phys. Rev. A 98, 042114 (2018).

[48] R. Okugawa and T. Yokoyama, Topological exceptional surfaces in non-Hermitian systems with parity-time and parity-particle-hole symmetries, Phys. Rev. B 99, 041202(R) (2019).

[49] K. Moors, A. A. Zyuzin, A. Yu. Zyuzin, R. P. Tiwari, and T. L. Schmidt, Disorder-driven exceptional lines and Fermi ribbons in tilted nodal-line semimetals, Phys. Rev. B 99, 041116(R) (2019)

[50] A. A. Zyuzin and A. Yu. Zyuzin, Flat band in disorder-driven non-Hermitian Weyl semimetals, Phys. Rev. B 97, 041203(R) (2018).

[51] T. Yoshida, R. Peters, N. Kawakami, and Y. Hatsugai, Symmetry-protected exceptional rings in two-dimensional correlated systems with chiral symmetry, Phys. Rev. B 99, 121101(R) (2019).

[52] J. Carlström, M. Stålhammar, J. C. Budich, and E. J. Bergholtz, Knotted non-Hermitian metals, Phys. Rev. B 99, 161115(R) (2019).

[53] E. J. Bergholtz and J. C. Budich, Non-Hermitian Weyl physics in topological insulator ferromagnet junctions, Phys. Rev. Res. 1, 012003 (2019).

[54] K. Kawabata, T. Bessho, and M. Sato, Classification of Exceptional Points and Non-Hermitian Topological Semimetals, Phys. Rev. Lett. 123, 066405 (2019).

[55] C. H. Lee and R. Thomale, Anatomy of skin modes and topology in non-Hermitian systems, Phys. Rev. B 99, 201103(R) (2019).

[56] T. Helbig, T. Hofmann, S. Imhof, M. Abdelghany, T. Kiessling, L. W. Molenkamp, C. Hua Lee, A. Szameit, M. Greiter, and R. Thomale, Observation of bulk boundary correspondence breakdown in topolectrical circuits, arXiv:1907.11562.

[57] C. H. Lee, G. Li, Y. Liu, T. Tai, R. Thomale, and X. Zhang, Tidal surface states as fingerprints of non-Hermitian nodal knot metals, arXiv:1812.02011.

[58] S. Lin, L. Jin, and Z. Song, Symmetry protected topological phases characterized by isolated exceptional points, Phys. Rev. B 99, 165148 (2019).

[59] L. Jin and Z. Song, Bulk-boundary correspondence in a nonHermitian system in one dimension with chiral inversion symmetry, Phys. Rev. B 99, 081103(R) (2019).

[60] D. Leykam, K. Y. Bliokh, C. Huang, Y. D. Chong, and F. Nori, Edge Modes, Degeneracies, and Topological Numbers in NonHermitian Systems, Phys. Rev. Lett. 118, 040401 (2017). 
[61] J. Y. Lee, J. Ahn, H. Zhou, and A. Vishwanath, Topological Correspondence between Hermitian and Non-Hermitian Systems: Anomalous Dynamics, Phys. Rev. Lett. 123, 206404 (2019).

[62] K. Kawabata, K. Shiozaki, M. Ueda, and M. Sato, Symmetry and Topology in Non-Hermitian Physics, Phys. Rev. X 9, 041015 (2019).

[63] H. Zhou and J. Y. Lee, Periodic table for topological bands with non-Hermitian symmetries, Phys. Rev. B 99, 235112 (2019).

[64] X. Wan, A. M. Turner, A. Vishwanath, and S. Y. Savrasov, Topological semimetal and Fermi-arc surface states in the electronic structure of pyrochlore iridates, Phys. Rev. B 83, 205101 (2011).

[65] X.-Q. Sun, C. C. Wojcik, S. Fan, and T. Bzdušek, Alice string in non-Hermitian systems, arXiv:1905.04338.

[66] A. Bouhon, R.-J. Slager, and T. Bzdušek, Non-abelian reciprocal braiding of Weyl points, arxiv:1907.10611.

[67] QuanSheng Wu, A. A. Soluyanov, and T. Bzdušek, Nonabelian band topology in noninteracting metals, Science $\mathbf{3 6 5}$, 1273 (2019).

[68] A. Tiwari and T. Bzdušek, Non-Abelian topology of nodal-line rings in $\mathcal{P} \mathcal{T}$-symmetric systems, arXiv:1903.00018.

[69] E. Prodan and H. Schulz-Baldes, Bulk and boundary invariants for complex topological insulators, Math. Phys. Studies (2016), .

[70] A. Hatcher, Algebraic Topology (Cambridge University Press, Cambridge, 2002).

[71] G. E. Volovik and V. P. Mineev, Investigation of singularities in superfluid $\mathrm{He}^{3}$ in liquid crystals by the homotopic topology methods, Zh. Eksp. Teor. Fiz. 72, 2256 (1977).

[72] N. D. Mermin, The topological theory of defects in ordered media, Rev. Mod. Phys. 51, 591 (1979).

[73] G. P. Alexander, B. Gin-ge Chen, E. A. Matsumoto, and R. D. Kamien, Colloquium: Disclination loops, point defects, and all that in nematic liquid crystals, Rev. Mod. Phys. 84, 497 (2012)

[74] J. Ahn, S. Park, and B.-J. Yang, Failure of Nielsen-Ninomiya Theorem and Fragile Topology in Two-Dimensional Systems with Space-Time Inversion Symmetry: Application to Twisted Bilayer Graphene at Magic Angle, Phys. Rev. X 9, 021013 (2019).

[75] J. Ahn, S. Park, D. Kim, Y. Kim, and B.-J. Yang, StiefelWhitney classes and topological phases in band theory, Chin. Phys. B 28, 117101 (2019).

[76] A. Bouhon, A. M. Black-Schaffer, and R.-J. Slager, Wilson loop approach to fragile topology of split elementary band representations and topological crystalline insulators with time-reversal symmetry, Phys. Rev. B 100, 195135 (2019).

[77] G. W. Whitehead, Elements of Homotopy Theory (Springer, New York, 1978).

[78] M. Arkowitz, Introduction to Homotopy Theory (Springer, New York, 2011).

[79] J. W. Milnor and J. D. Stasheff, Characteristic classes, Ann. Math. Stud. 76 (1975).

[80] J. E. Moore, Y. Ran, and X.-G. Wen, Topological Surface States in Three-Dimensional Magnetic Insulators, Phys. Rev. Lett. 101, 186805 (2008).
[81] D.-L. Deng, S.-T. Wang, C. Shen, and L.-M. Duan, Hopf insulators and their topologically protected surface states, Phys. Rev. B 88, 201105(R) (2013).

[82] R. Kennedy, Topological Hopf-Chern insulators and the Hopf superconductor, Phys. Rev. B 94, 035137 (2016).

[83] A. Alexandradinata, A. Nelson, and A. A. Soluyanov, The actually robust surface signature of a Hopf insulator: Bulk-toboundary flow of Berry curvature beyond the anomaly inflow paradigm, arXiv:1910.10717.

[84] S. Lang, Algebra (Springer, Berlin, 2002).

[85] P. Crooks, Complex adjoint orbits in Lie theory and geometry, arXiv:1703.03390.

[86] G. J. Ellis, Homotopy classification the J.H.C. Whitehead way, Exposition. Math. 6, 97 (1988).

[87] J. P. Ang and A. Prakash, Higher categorical groups and the classification of topological defects and textures, arXiv:1810.12965.

[88] D. Xiao, M.-C. Chang, and Q. Niu, Berry phase effects on electronic properties, Rev. Mod. Phys. 82, 1959 (2010).

[89] H. C. Po, H. Watanabe, and A. Vishwanath, Fragile Topology and Wannier Obstructions, Phys. Rev. Lett. 121, 126402 (2018).

[90] N. Johansson and E. Sjöqvist, Optimal Topological Test for Degeneracies of Real Hamiltonians, Phys. Rev. Lett. 92, 060406 (2004).

[91] T. Bzdušek and M. Sigrist, Robust doubly charged nodal lines and nodal surfaces in centrosymmetric systems, Phys. Rev. B 96, 155105 (2017).

[92] C. Fang, H. Weng, X. Dai, and Z. Fang, Topological nodal line semimetals, Chin. Phys. B 25, 117106 (2016).

[93] Y. X. Zhao and Y. Lu, $\mathcal{P} \mathcal{T}$-Symmetric Real Dirac Fermions and Semimetals, Phys. Rev. Lett. 118, 056401 (2017).

[94] Z. Song, Z. Wang, W. Shi, G. Li, C. Fang, and B. A. Bernevig, All Magic Angles in Twisted Bilayer Graphene are Topological, Phys. Rev. Lett. 123, 036401 (2019).

[95] H. C. Po, L. Zou, T. Senthil, and A. Vishwanath, Faithful tightbinding models and fragile topology of magic-angle bilayer graphene, Phys. Rev. B 99, 195455 (2019).

[96] R. Bistritzer and A. H. MacDonald, Moiré bands in twisted double-layer graphene, Proc. Natl. Acad. Sci. U. S. A. 108, 12233 (2011).

[97] Y. Cao, V. Fatemi, S. Fang, K. Watanabe, T. Taniguchi, E. Kaxiras, and P. Jarillo-Herrero, Unconventional superconductivity in magic-angle graphene superlattices, Nature (London) 556, 43 (2018).

[98] Y. Cao, V. Fatemi, A. Demir, S. Fang, S. L. Tomarken, J. Y. Luo, J. D. Sanchez-Yamagishi, K. Watanabe, T. Taniguchi, E. Kaxiras, R. C. Ashoori, and P. Jarillo-Herrero, Correlated insulator behavior at half-filling in magic-angle graphene superlattices, Nature (London) 556, 80 (2018).

[99] A. A. Soluyanov and D. Vanderbilt, Wannier representation of $\mathbb{Z}_{2}$ topological insulators, Phys. Rev. B 83, 035108 (2011).

[100] D. Gresch, G. Autès, O. V. Yazyev, M. Troyer, D. Vanderbilt, B. A. Bernevig, and A. A. Soluyanov, Z2Pack: Numerical implementation of hybrid Wannier centers for identifying topological materials, Phys. Rev. B 95, 075146 (2017).

[101] J. Ahn, D. Kim, K. Youngkuk, and B.-J. Y. Yang, Band Topology and Linking Structure of Nodal Line Semimetals 
with $\mathbb{Z}_{2}$ Monopole Charges, Phys. Rev. Lett. 121, 106403 (2018).

[102] G. Carlsson, Topology and data, Bull. Am. Math. Soc 46, 255 (2009).
[103] L. Yuan, Q. Lin, M. Xiao, and S. Fan, Synthetic dimension in photonics, Optica 5, 1396 (2018).

[104] Z. Li and R. S. K. Mong, Homotopical classification of nonHermitian band structures, arXiv:1911.02697. 